\title{
Habitable Zone Lifetimes of Exoplanets around Main Sequence Stars
}

\author{
Andrew J. Rushby, Mark W. Claire, ${ }^{1,2}$ Hugh Osborn, ${ }^{3}$ and Andrew J. Watson ${ }^{1,4}$
}

\begin{abstract}
The potential habitability of newly discovered exoplanets is initially assessed by determining whether their orbits fall within the circumstellar habitable zone of their star. However, the habitable zone (HZ) is not static in time or space, and its boundaries migrate outward at a rate proportional to the increase in luminosity of a star undergoing stellar evolution, possibly including or excluding planets over the course of the star's main sequence lifetime. We describe the time that a planet spends within the HZ as its "habitable zone lifetime." The HZ lifetime of a planet has strong astrobiological implications and is especially important when considering the evolution of complex life, which is likely to require a longer residence time within the HZ. Here, we present results from a simple model built to investigate the evolution of the "classic" HZ over time, while also providing estimates for the evolution of stellar luminosity over time in order to develop a "hybrid" HZ model. These models return estimates for the HZ lifetimes of Earth and 7 confirmed HZ exoplanets and 27 unconfirmed Kepler candidates. The HZ lifetime for Earth ranges between 6.29 and $7.79 \times 10^{9}$ years (Gyr). The 7 exoplanets fall in a range between $\sim 1$ and $54.72 \mathrm{Gyr}$, while the 27 Kepler candidate planets' HZ lifetimes range between 0.43 and 18.8 Gyr. Our results show that exoplanet HD 85512b is no longer within the HZ, assuming it has an Earth analog atmosphere. The HZ lifetime should be considered in future models of planetary habitability as setting an upper limit on the lifetime of any potential exoplanetary biosphere, and also for identifying planets of high astrobiological potential for continued observational or modeling campaigns. Key Words: Exoplanet habitability metrics-Continuously habitable zone-Stellar evolution-Planetary habitability. Astrobiology 13, 833-849.
\end{abstract}

\section{Introduction}

$\mathbf{U}$ NDERSTANDING the nature and distribution of planets and moons throughout the Galaxy that are able to sustain life is a major research theme identified by the NASA Astrobiology Roadmap (Des Marais et al., 2008). The study of planetary habitability encompasses research and methodological approaches from a wide variety of disciplines within the physical and environmental sciences. Reflecting this trend, a number of multiparameter habitability indices, most closely analogous to those employed for ecological surveys, have been recently developed (e.g., Schulze-Makuch et al., 2011; Planetary Habitability Laboratory, 2012) in an attempt to provide a quantitative framework for the assessment of the habitability of the ever-growing catalogue of extrasolar planets. Habitability metrics are useful diagnostic and comparative tools for investigating the potential for extrasolar planets to host life, and they allow for classification and comparison between exoplanets and Earth. Crucially, they are also valuable for prioritizing interesting planetary candidates for future or continued observational campaigns. However, most indices described to date neglect the evolving nature of planetary habitability over astronomical and geological time.

The location of a planet within the circumstellar habitable zone is a measure that has been adopted to determine planetary habitability to first order (Kasting et al., 1993; Selsis et al., 2007). The habitable zone (HZ) forms a fundamental component of many contemporary habitability metrics, and our understanding of the distribution and formation of habitable planetary environments strongly depends on the criteria used to set the boundaries of the HZ. Despite the integral role of planetary habitability in astrobiological research, the $\mathrm{HZ}$ remains a relatively rigid quantification of an inherently complex, multifaceted problem.

The HZ describes the circumstellar distance at which surface temperatures allow liquid water to be present on the

\footnotetext{
${ }^{1}$ School of Environmental Sciences, University of East Anglia, Norwich, UK.

${ }^{2}$ Department of Earth and Environmental Sciences, University of St. Andrews, St. Andrews, UK.

${ }^{3}$ Department of Earth Sciences, University College London, London, UK.

${ }^{4}$ Department of Geography, University of Exeter, Exeter, UK.
} 


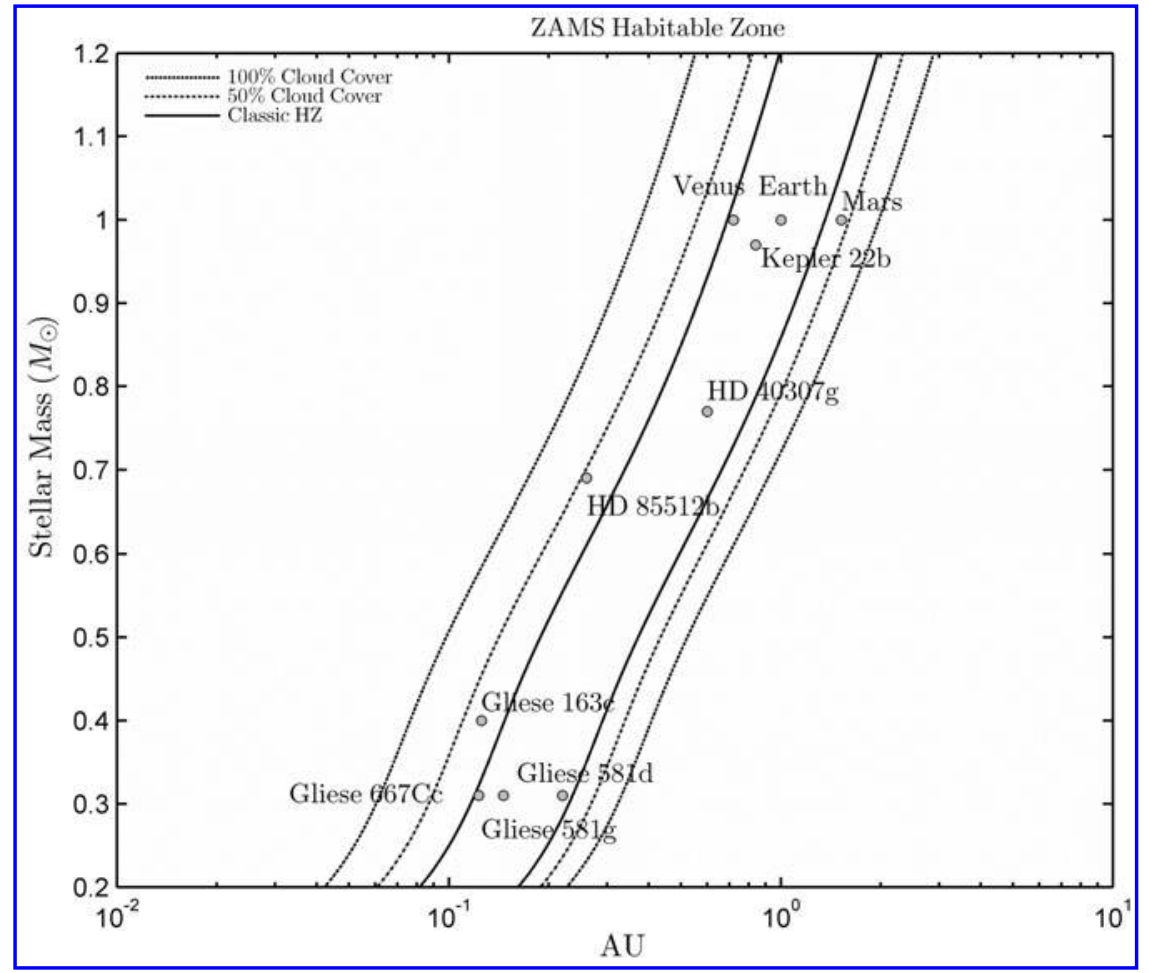

FIG. 1. The position of the HZ at the ZAMS stage of stellar evolution as a function of stellar mass. Earth and seven confirmed HZ exoplanets are also plotted, along with Mars and Venus as proxies for distance in the Solar System. The outer dashed lines represent a 100\% cloud cover scenario, the dotted lines $50 \%$, and the solid lines the "classic" HZ.

planet's surface, assuming variable $\mathrm{H}_{2} \mathrm{O} / \mathrm{CO}_{2} / \mathrm{CH}_{4}$ greenhouse forcings. The circumstellar $\mathrm{HZ}$ concept is based on Earth analog terrestrial planets that exhibit dynamic lithospheric tectonic activity with magnetospheres; high $(\sim 80 \%)$ humidity; and 1 bar atmospheres of $\mathrm{N}_{2}, \mathrm{H}_{2} \mathrm{O}$, and $\mathrm{CO}_{2}$ (Kasting et al., 1993; Raymond et al., 2007). The HZ has a minimum and maximum extent, forming inner (closer to the star) and outer boundaries that are set in part by biogeo- chemical climate feedback mechanisms and stellar luminosity (see Figs. 1 and 2).

The minimum extent, or inner boundary, is set by the water-vapor feedback mechanism, and the maximum, outer boundary is controlled by the dynamics of the carbon-silicate cycle (Kasting et al., 1993; Tarter et al., 2007). The water-vapor feedback describes a positive ("runaway") feedback mechanism that operates on a planet with liquid water at its

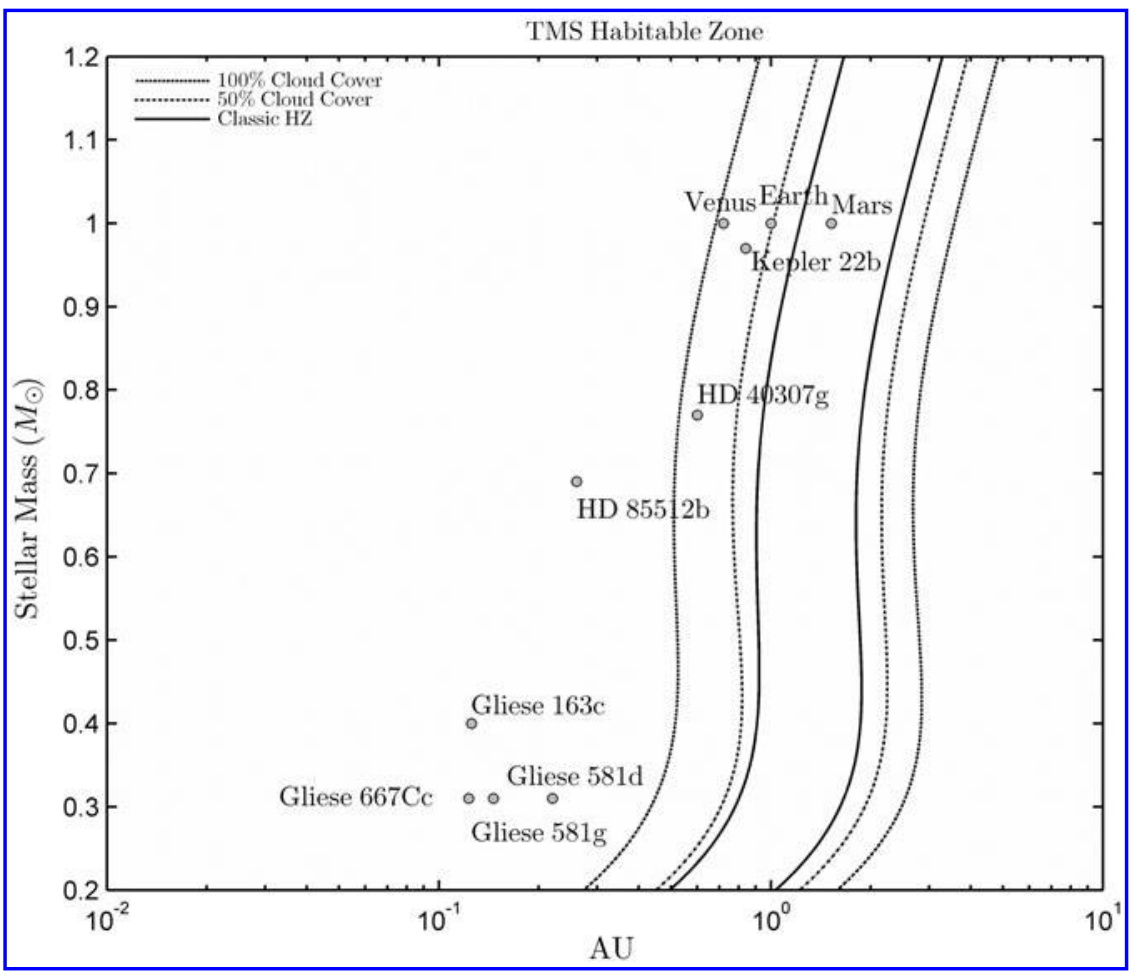

FIG. 2. The position of the $\mathrm{HZ}$ at the TMS stage of stellar evolution as a function of stellar mass. 
surface, experiencing high rates of evaporation and increasing humidity. The resulting increase in temperature serves to further accelerate evaporation, eventually resulting in the irreversible evaporation of the ocean into the atmosphere (Kasting, 1988; Goldblatt and Watson, 2012). The outer boundary is set at the temperature at which $\mathrm{CO}_{2}$ clouds first start to form in the atmosphere of a planet with a fixed $273 \mathrm{~K}$ surface temperature in the "conservative" model used by Kasting et al. (1993). The radiative effects of these clouds are complex and likely wavelength-dependent, and their effect on climate has not yet been well constrained (Colaprete and Toon, 2003; Kitzmann et al., 2010; Goldblatt and Zahnle, 2011; Joshi and Haberle, 2012; Zsom et al., 2012). Selsis et al. (2007) attempted to reconcile this effect by considering these clouds as purely reflective, albedo features. However, it is also possible that $\mathrm{CO}_{2}$ clouds may have the additional effect of reflecting outgoing thermal radiation back to the surface of the planet, contributing to a net warming effect (von Bloh et al., 2007). More-sophisticated climate modeling approaches are required to fully constrain the complex effect that clouds, in atmospheres of varying compositions, will have on planetary climate under different stellar environments.

The carbon-silicate cycle is fundamental in determining the $\mathrm{CO}_{2}$ mixing ratio of the atmosphere, and active plate tectonics and volcanism are necessary for this biogeochemical cycle to operate (Sleep and Zahnle, 2001). However, it is possible that more massive "super-Earths" may exhibit an entirely different mode of thermal evolution due to the fact that they are likely to have internal pressures tens of times higher than those found in Earth's interior, large viscosities, and higher melting temperatures (Kite et al., 2009; Stamenković et al., 2012). Some studies suggest that the greater shear stresses and thinner plates thought to be associated with planets of higher masses will favor subduction by decreasing the overall resistance to plate motion (Valencia et al., 2007). Others maintain that a "stagnant lid" or episodic tectonic regime may be a more realistic assumption because of a modeled reduction in the ratio of driving to resistive forces and increased fault strength under high gravity ( $\mathrm{O}^{\prime}$ Neill and Lenardic, 2007). Further modeling and eventual observation of exoplanetary atmospheres and/or interiors will settle this debate with time.

The existence of surficial liquid water is thought to be a fundamental prerequisite for the emergence and continued survival of life because of its important role as a solvent for biochemical reactions. In addition, water is available in appreciable amounts across the Galaxy and in interstellar clouds (Lammer et al., 2009). Water is also present in significant quantities in comets and protoplanetary bodies; delivery of the water reservoir of Earth is thought to have been via impact with similar, water-rich objects during the early, violent stages of planetary accretion (Raymond et al., 2007; Lammer et al., 2009). The existence of liquid water also requires planetary temperatures to be within the range expected to be optimal for life.

\subsection{On the definition of the "habitable zone"}

Throughout this paper, we use the seminal HZ study of Kasting et al. (1993) and the Selsis et al. (2007) extension of this work, but it should be recognized that this definition is one of many and is applicable only under the assumptions of
"Earth-like" planetary mass and composition, tectonics, and atmospheric pressure and composition. The planets in our sample are unlikely to conform to all these conditions, and these restrictions should be considered when interpreting our results. To date, a true Earth analog planet has not been detected.

The concept of a "habitable zone" is continuously evolving, from the original form to subsurface and tidal HZs (Heller et al., 2011; Barnes et al., 2013), dry planet HZs (Abe et al., 2011), and even possible galactic HZs (Lineweaver et al., 2004). Pierrehumbert and Gaidos (2011) provided an alternative definition of the HZ that extends to larger distances than the Earth analog HZ of Kasting et al. (1993) for exoplanets with primordial hydrogen-rich atmospheres, and the possibility of ejected "rogue" planets in interstellar space has also been considered (Abbot and Switzer, 2011). A detailed summary of the factors affecting exoplanetary habitability can be found in Seager (2013).

Kopparapu et al. (2013) made further revisions to the Kasting et al. (1993) model, using a 1-D radiative-convective model, and results suggest an inner boundary of the $\mathrm{HZ}$ farther from the star. We expect that many more HZ models of varying complexity will be developed in the future as understanding of this area advances; our aim is to provide a model framework that can be applied to any radiative HZ model by coupling luminosity evolution with HZ boundary transition rates to return a $\mathrm{HZ}$ lifetime estimate. Additionally, we focus on stellar-based HZ estimations because they can provide a certain degree of observability that other parameterizations cannot; direct measurements of stellar luminosity can be made, and theories regarding stellar evolution and effective temperature are well developed in the astronomical literature.

While the definition of the HZ boundary will continue to be refined with further research, these uncertainties are not the focus of this study. Regardless of the definition used to identify their particular position in space, the boundaries of a radiative $\mathrm{HZ}$ are unequivocally not temporally static, but rather they vary over astronomical time as a function of the secular increase in luminosity of a star undergoing stellar evolution (Kasting et al., 1993; Claire et al., 2012). As a star evolves through the main sequence, its luminosity increases, and the boundaries of the HZ "migrate" outward at a rate proportional to this increase. The effect that this well-known and uncontroversial process has on the evolution of the HZ has not been well quantified, and the accelerating discovery of habitable planets necessitates its estimation.

As planets move out of, or into, the $\mathrm{HZ}$ over the course of a star's main sequence lifetime, the time that any particular planet spends within the $\mathrm{HZ}$ can then be defined as its habitable period or "habitable zone lifetime." The HZ lifetime of a planet is especially important when considering the potential for the evolution of complex life, which is likely to require a longer residence time within the $\mathrm{HZ}$ (Watson, 2008).

Toward the end of a planet's HZ lifetime, steadily increasing stellar luminosity is likely to result in a runaway greenhouse event, which would represent a catastrophic and terminal extinction event for any surface biosphere present on the planet (Pierrehumbert, 2010; Goldblatt and Watson, 2012). The end of a planet's HZ lifetime and the end of its host star's main sequence lifetime do not necessarily have to 
Table 1. Elements of the Fitting Matrices for $0.08 \geq \operatorname{Mass}\left(M_{\odot}\right)<0.45$ And $0>\tau($ Grr $)<12.6$

\begin{tabular}{lccccc}
\hline$p_{00}$ & $p_{10}$ & $p_{01}$ & $p_{20}$ & $p_{11}$ & $p_{02}$ \\
-0.0009502 & 0.0001695 & -0.001583 & $-4.403 \times 10^{-6}$ & -0.003114 & 0.2553 \\
$p_{21}$ & $p_{12}$ & $p_{03}$ & $p_{22}$ & $p_{13}$ & -0.01762 \\
$6.739 \times 10^{-5}$ & 0.01549 & -0.6876 & -0.0002243 & 0.9136 \\
\hline
\end{tabular}

coincide. For example, the Sun is likely to become a red giant several billion years after the likely cessation of habitable conditions on Earth. Estimations for the termination of Earth's biosphere have been calculated by other workers who used models optimized for this planet; we attempt a more general, flexible, and stellar-centered approach that can be applied to both Earth and to extrasolar Earth analogues (Caldeira and Kasting, 1992; Franck et al., 2000; Goldblatt and Watson, 2012; O'Malley-James et al., 2012).

\section{Methods}

The basis of this HZ lifetime model is derived from an estimated "transition rate" of the dynamic HZ boundaries over the course of stellar evolution, from the zero-age main sequence (ZAMS) (Fig. 1) to the termination of the main sequence (TMS) (Fig. 2) configurations. Main sequence lifetime is denoted as $\tau$ in the following sections, and stellar mass normalized to the Sun, where $1 M_{\odot}=1$ solar mass.

\subsection{Luminosity as a function of stellar mass and time}

The rates of transition of the HZ's boundaries are proportional to the change in stellar luminosity over the star's lifetime. In the first iteration of this model, this secular increase in stellar luminosity over time (ZAMS to TMS) was assumed to be linear to first order. While a relatively justifiable assumption for a model of this complexity, we felt that more realistic tracks of luminosity over time were required to better capture the nonlinear nature of early and late stellar evolution. Therefore, we used a multi-dimensional ( 3 by 5 ) polynomial fit to determine luminosity $(L)$ as a function of both mass $(M)$ and time $(\tau)$. Luminosity is measured in solar units, where $L=1$ is equivalent to the current luminosity of the Sun, given as $3.846 \times 10^{26} \mathrm{~W}$.

Baraffe et al. (1998) created stellar evolutionary models across the $0.075-1.0 M_{\odot}$ and an age range spanning 0-12.6 Gyr. These models produced values for effective temperature $\left(T_{\text {eff }}\right)$, surface gravity $(\log g)$, and absolute bolometric magnitude $\left(M_{\text {bol }}\right)$. Although the authors varied metallicity ([M/ $\mathrm{H}]$ ) and helium mass fraction $(Y)$, we held $[\mathrm{M} / \mathrm{H}]$ constant at solar values (0.0) and $Y$ at 0.275 .

Luminosity was calculated by using both the $\log g$ and bolometric magnitude values:

$$
L_{M_{\mathrm{bol}}}=10 \frac{\left(4.75-M_{\mathrm{bol}}\right)}{2.5}
$$

$$
L_{\log g}=4 \pi\left(\frac{G M}{10^{\log g}}\right) \sigma T^{4}
$$

These values agreed to within $2 \%$, and a mean luminosity was calculated by taking an average to provide $L_{\text {mean }}$.

The Baraffe model begins pre-main sequence where the continued collapse as set out by the Henyey and Hayashi tracks causes a decrease in luminosity. This is especially prominent in low-mass stars, which do not settle onto the main sequence for up to 1 Gyr. By estimating the point at which the star's luminosity became stable, we removed this initial decrease in order to fit curves to the main sequence regions.

We plotted polynomials to the data, but initial results represented the low-mass regions of the data poorly, so the data was split by mass at $0.45 M_{\odot}$ (Tables 1 and 2):

$$
\begin{aligned}
L(M, \tau)= & p_{00}+p_{10} \tau+p_{01} M+p_{20} \tau^{2}+p_{11} \tau M+p_{02} M^{2} \\
& +p_{21} \tau^{2} M+p_{12} \tau M^{2}+p_{03} M^{3}+p_{22} \tau^{2} M^{2} \\
& +p_{13} \tau M^{3}+p_{04} M^{4} \\
L(M, \tau)= & p_{00}+p_{10} \tau+p_{20} \tau^{2}+p_{11} \tau M+p_{02} M^{2}+p_{21} \tau^{2} M \\
& +p_{12} \tau M^{2}+p_{03} M^{3}+p_{22} \tau^{2} M^{2}+p_{13} \tau M^{3}+p_{04} M^{4} \\
& +p_{23} \tau^{2} M^{3}+p_{14} \tau M^{4}+p_{05} M^{5}
\end{aligned}
$$

The fits were corrected to conform to $1 M_{\odot}=1 L_{\odot}$ at $\tau=4.54$ Gyr and were linearly extended to $L_{\mathrm{TMS}}$ with figures from Guo et al. (2009), as shown in Fig. 3 and Table 3.

To approximate main sequence lifetime $(\tau)$ in billions of years as a function of stellar mass, we used the 2012 online update to the Dartmouth Stellar Evolution Database to obtain isochrones for low-mass stars (using solar parameters) at 4.56, 10, and 15 Gyr (Dotter et al., 2008). As expected for the lower-mass stars with minimal luminosity evolution, the mass-luminosity relations for each isochrone were nearly identical and are fitted by

$$
\begin{aligned}
& L(M)=0.1155\left(\frac{M}{M_{\odot}}\right)^{1.9513}\left(>0.08<0.42 M_{\odot}\right) \\
& L(M)=0.9455\left(\frac{M}{M_{\odot}}\right)^{4.7772}\left(>0.42<0.8 M_{\odot}\right)
\end{aligned}
$$

Table 2. Elements of the Fitting Matrices for $0.45 \geq \operatorname{Mass}\left(M_{\odot}\right)<1.0$ AND $0>\tau($ Grr $)<12.6$

\begin{tabular}{lcccccc}
\hline$p_{00}$ & $p_{10}$ & $p_{01}$ & $p_{20}$ & $p_{11}$ & $p_{02}$ & $p_{21}$ \\
-2.245 & 0.7376 & 16.03 & -0.02348 & -4.596 & -44.2 & 0.1212 \\
$p_{03}$ & $p_{22}$ & $p_{13}$ & $p_{04}$ & $p_{23}$ & $p_{14}$ & 10.5 \\
59.23 & -0.2047 & -10.43 & -38.59 & 0.1132 & 3.82 & 10.46 \\
\hline
\end{tabular}


FIG. 3. Main sequence luminosity as a function of stellar mass (at $0.1 \mathrm{M}_{\odot}$ intervals) and time. From 1 to $\sim 12.6 \mathrm{Gyr}$, luminosity evolution was modeled by using Eqs. 3 and 4 (above) and extended linearly to $L_{\mathrm{TMS}}$ at $\tau$.

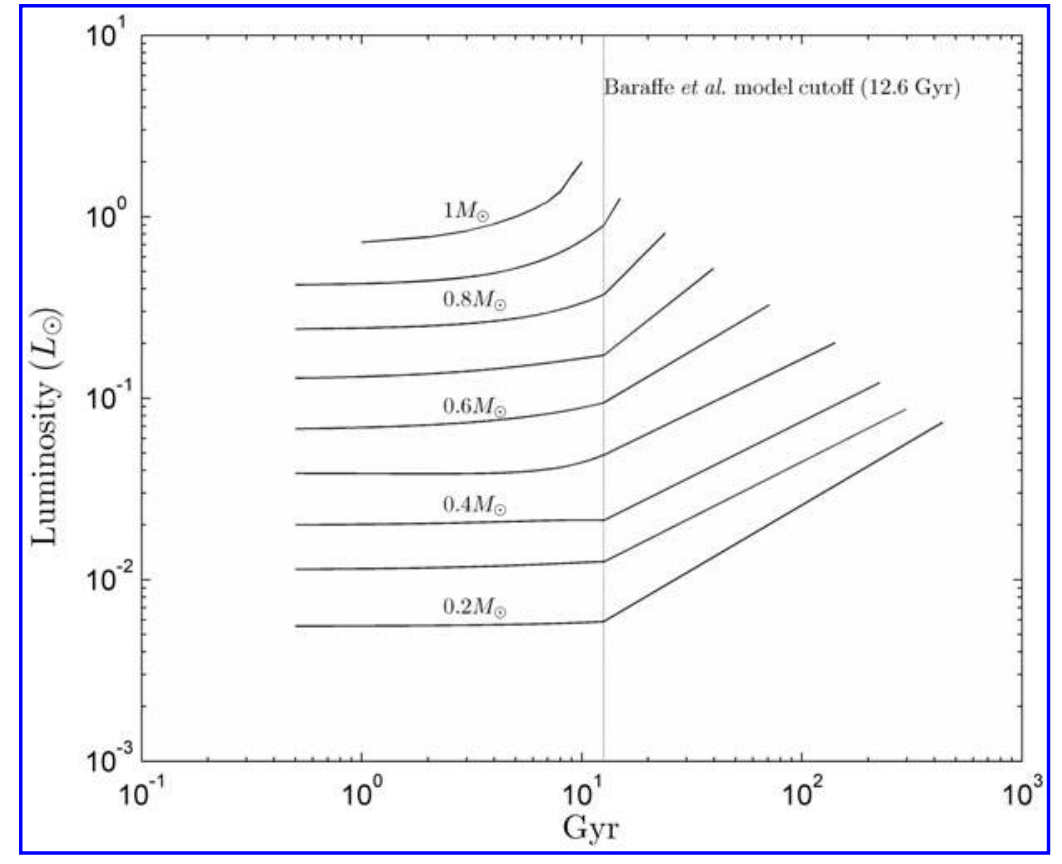

where we have used the 10 Gyr isochrones to make the fits. The fits for $\mathrm{K}$ class stars $\left(0.42-0.8 M_{\odot}\right)$ provided here are the result of the normalization of the multiplicative factors after the initial fitting to ensure that the parameterizations remain continuous at the overlaps between the mass classes. For higher-mass stars $\left(0.8-1.2 \mathrm{M}_{\odot}\right)$, we have adopted a simple scaling (Nebojsa, 2004):

$$
L(M)=\left(\frac{M}{M_{\odot}}\right)^{4}\left(>0.8<1.2 M_{\odot}\right)
$$

We can then compute main sequence lifetimes in the zerothorder manner:

$$
\tau=10.9 \times\left(\frac{M}{L}\right)
$$

TAble 3. Luminosity at 12 Gyr ( $\left.L_{12 \mathrm{Gyr}}\right)$, Luminosity at the END of the Main SEquence ( $\left.L_{\text {TMS }}\right)$, ANd Main Sequence Lifetime $(\tau)$ for StaRs BETWEEN 0.2 AND $1.2 M_{\odot}$

\begin{tabular}{lccc}
\hline Mass $\left(\mathrm{M}_{\odot}\right)$ & $\mathrm{L}_{12 \text { Gyr }}\left(\mathrm{L}_{\odot}\right)^{\mathrm{a}}$ & $\mathrm{L}_{T M S}\left(\mathrm{~L}_{\odot}\right)^{\mathrm{b}}$ & $\tau(\mathrm{Gyr})$ \\
\hline 0.2 & 0.0051099 & 0.073602 & 436.3 \\
0.25 & 0.0081265 & 0.081851 & 352.8 \\
0.3 & 0.011746 & 0.086928 & 296.7 \\
0.35 & 0.015975 & 0.098572 & 256.2 \\
0.4 & 0.020817 & 0.12201 & 225.6 \\
0.45 & 0.025177 & 0.15688 & 210.4 \\
0.5 & 0.038696 & 0.20191 & 141.3 \\
0.6 & 0.070764 & 0.3262 & 71 \\
0.7 & 0.13689 & 0.51758 & 39.6 \\
0.8 & 0.3292 & 0.813 & 23.9 \\
0.9 & 0.8181 & 1.2576 & 14.9 \\
1 & $\mathrm{c}$ & 2.28 & 10.9 \\
1.1 & $\mathrm{c}$ & 3.1 & 8.2 \\
1.2 & $\mathrm{c}$ & 4.25 & 6.3 \\
\hline
\end{tabular}

a Baraffe et al. (1998); ${ }^{\mathrm{b}} \mathrm{Guo}$ et al. (2009); 'Beyond model limits. where $\tau$ is in billions of years, 10.9 is the solar main sequence lifetime, and $L(M)$ are in the age ranges above (Sackmann et al., 1993).

These estimates of main sequence lifetimes are shown in Table 3 and Fig. 4 . For the lowest-mass stars $\left(0.08-0.25 M_{\odot}\right)$, this procedure results in main sequence lifetimes from 350 to 1000 Gyr and returns comparable values to those of Laughlin (1997), within an order of magnitude. Our results are likely to be less robust as they are not direct computations from observational data, but we used our fitted main sequence lifetimes for consistency as there are no comparable calculations of main sequence lifetimes for stars between 0.25 and $0.5 M_{\odot}$.

Table 3 provides values of luminosity at 12 Gyr $\left(L_{12 \mathrm{Gyr}}\right)$, which represents the final data from the Baraffe et al. (1998)

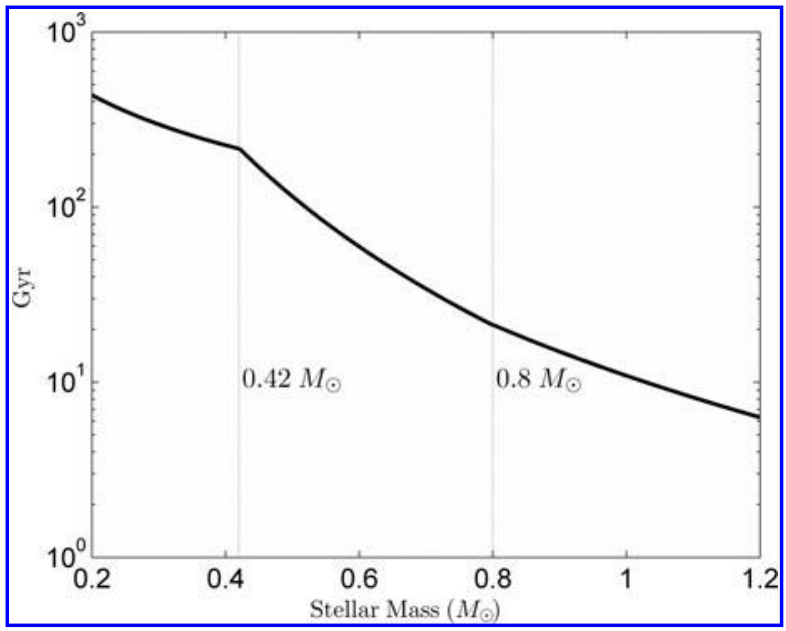

FIG. 4. Main sequence age as a function of stellar mass for stars between 0.2 and $1.2 M_{\odot}$. Between 0.08 and $0.42 M_{\odot}$, see Eq. 5. Between 0.42 and $0.8 M_{\odot}$ Eq. 6 is used, and Eq. 7 governs the relationship between 0.8 and $1.2 M_{\odot}$. 
stellar evolution model, luminosity at the end of the main sequence ( $\left.L_{\mathrm{TMS}}\right)$ from Guo et al. (2009), and main sequence lifetime $(\tau)$, computed as shown above.

Between 0.2 and $0.9 M_{\odot}$, the Baraffe data set allows for polynomial fits of $L(\tau, M)$ between $\sim 0.5$ and 12 Gyr. Solar mass $\left(1 M_{\odot}\right)$ fits extend to 8 Gyr. However, many low-mass stars have main sequence lifetimes an order of magnitude greater than this upper limit. Accordingly, we used the initial polynomial fits between $0.5<\tau>12\left(0.5<\tau>8\right.$ Gyr for $\left.1 M_{\odot}\right)$ and assumed a linear progression to the final $L_{\text {TMS }}$ stage to produce a "hybrid" track of $L$, where $L_{\mathrm{TMS}}$ is given by Guo et al. (2009) and $\tau_{\mathrm{TMS}}$ is given by Eqs. 5-8, normalized to $\tau_{\text {sun }}=10.9$ Gyr.

\subsection{The habitable zone}

The inner and outer boundaries of the radiative $\mathrm{HZ}$ are set by the limit of the water-vapor feedback mechanism and the carbon-silicate cycle $/ \mathrm{CO}_{2}$ condensation limit, respectively. The inner boundary of the climatic $\mathrm{HZ}$ for a star with a given luminosity and effective stellar temperature, $T_{\text {eff }}$ (in kelvin), can be calculated according to the work of Jones et al. (2006):

$$
\mathrm{HZ}_{\text {inner }}=\left(\frac{L}{S_{\text {in }}\left(T_{\text {eff }}\right)}\right)^{\frac{1}{2}}
$$

where the critical stellar flux at the inner boundary of the $\mathrm{HZ}, S_{\text {in }}\left(T_{\text {eff }}\right)$, dependent on the $T_{\text {eff }}$ of the star, is given as a second-order polynomial:

$$
S_{\text {in }}\left(T_{\text {eff }}\right)=\left(4.190 \times 10^{-8} T_{\text {eff }}^{2}\right)-\left(2.139 \times 10^{-4} T_{\text {eff }}\right)+1.296
$$

Luminosity, stellar radius, and effective temperature are related according to the Stefan-Boltzmann law:

$$
L=4 \pi R^{2} \sigma T_{\text {eff }}^{4}
$$

Where $\sigma$ is the Stefan-Boltzmann constant, given as $5.67 \times 10^{-8} \mathrm{~J} \mathrm{~s}^{-1} \mathrm{~m}^{-2} \mathrm{~K}^{-4}$, and $R$ is stellar radius.

Jones et al. (2006) went on to define the outer limit of the $\mathrm{HZ}$ as

$$
\mathrm{HZ}_{\text {outer }}=\left(\frac{L}{S_{\text {outer }}\left(T_{\text {eff }}\right)}\right)^{\frac{1}{2}}
$$

The critical stellar flux at the outer boundary of the $\mathrm{HZ}, S_{\text {out }}$ $\left(T_{\text {eff }}\right)$, is given by

$$
S_{\text {out }}\left(T_{\text {eff }}\right)=\left(6.190 \times 10^{-9} T_{\text {eff }}^{2}\right)-\left(1.139 \times 10^{-5} T_{\text {eff }}\right)+0.2341
$$

To ascertain the luminosity of a given star at ZAMS and TMS, it is necessary to invoke a number of formulae and fitting coefficients, taken from Table 1 of Guo et al. (2009).

Due to the relative uncertainties regarding the mechanisms that control the actual extent of the HZ, especially the ambiguous hot zone/inner boundary transition, we have also used the HZ boundary estimations outlined by Selsis et al. (2007) to consider the effect of $50 \%$ and $100 \%$ cloud cover regimes, given by

$$
\begin{gathered}
\mathrm{HZ}_{\text {inner }}=\left(\mathrm{HZ}_{\text {inner } \odot}-a_{\text {in }} T_{*}^{2}-b_{\text {in }} T_{*}^{2}\right)\left(\frac{L}{L_{\odot}}\right)^{\frac{1}{2}} \\
\mathrm{HZ}_{\text {outer }}=\left(\mathrm{HZ}_{\text {outer } \odot}-a_{\text {out }} T_{*}^{2}-b_{\text {out }} T_{*}^{2}\right)\left(\frac{L}{L_{\odot}}\right)^{\frac{1}{2}}
\end{gathered}
$$

Here, $a_{\text {in }}=2.7619 \times 10^{-5}, b_{\text {in }}=3.8095 \times 10^{-9}, a_{\text {out }}=1.3786 \times 10^{-4}$, $b_{\text {out }}=1.4286 \times 10^{-9}$, and $T_{*}=\left(T_{\text {eff }}-5700 \mathrm{~K}\right)$. Fifty percent cloud cover boundaries are set by the authors as $\mathrm{HZ}_{\text {inner } \odot}=0.68$ to $0.76 \mathrm{AU}$ (mean: $0.72 \mathrm{AU}$ ) and $\mathrm{HZ}_{\text {outer } \odot}=1.67$ AU. The boundary for a $100 \%$ cloud cover $\mathrm{HZ}$ is $\mathrm{HZ}_{\text {inner } \odot}=0.46$ to $0.51 \mathrm{AU}($ mean $=0.49 \mathrm{AU})$ and $\mathrm{HZ}_{\text {inner } \odot}=2.4$ AU. Equations 14 and 15 provide a similar result to Eqs. 9 and 12 under a $\sim 30 \%$ cloud cover scenario, but for the purposes of this study and for consistency, we maintain the classic HZ as defined by Eqs. 9 and 12, and utilize Eqs. 8 and 9 for testing varying cloud cover regimes only (Selsis et al., 2007). We note, however, that the "cloud cover" included in this model is justifiably parameterized as an albedo (i.e., reflective) feature and not the result of modeling the multiparameter effect of water vapor or $\mathrm{CO}_{2}$ clouds on climate over long timescales. We adopt the nomenclature of Lammer et al. (2009) and designate planets within these near-inner or outer boundary zones of the HZ as Class II habitable planets. Class I planets are those that fall within the classic HZ, as given in Eqs. 9 and 12.

\subsection{Habitable zone boundary transition rates $(\mu)$}

As discussed in the previous section, two estimations of the change in $L$ over time can be used to model the change in the HZ over time: one hybrid fit [polynomial fit for $L(M, \tau)$ to $L_{12 \mathrm{Gyr}}$ coupled with a linear extrapolation to $\left.L_{\mathrm{TMS}}\right]$ and the other purely linear (simply $L_{\mathrm{TMS}}-L_{\mathrm{ZAMS}} / \tau$ ).

It is then possible to attain a "transition rate" of the inner and outer boundaries (denoted by $\mu_{\text {inner }}$ and $\mu_{\text {outer }}$ respectively) of the $\mathrm{HZ}$ for main sequence stars between 0.2 and 1.2 solar masses $\left(M_{\odot}\right)$ by using a simple linear relationship:

$$
\begin{aligned}
& \mu_{\text {inner }}=\frac{\left(\mathrm{HZ}_{\text {inner }}^{\mathrm{TMS}}-\mathrm{HZ}_{\text {inner }}^{\mathrm{ZAMS}}\right)}{\tau} \\
& \mu_{\text {outer }}=\frac{\left(\mathrm{HZ}_{\text {outer }}^{\mathrm{TMS}}-\mathrm{HZ}_{\text {outer }}^{\mathrm{ZAMS}}\right)}{\tau}
\end{aligned}
$$

Our results are presented in astronomical units per billion years $\left(\mathrm{AU} \mathrm{Gyr}{ }^{-1}\right.$ ) in Fig. 3. The terms $\mathrm{HZ}_{\text {inner }}^{\mathrm{TMS}}$ and $\mathrm{HZ}_{\text {inner }}^{\mathrm{ZAMS}}$ describe the distance (in $\mathrm{AU}$ ) of the position of the inner boundary of the HZ at the TMS and the ZAMS stages of stellar evolution and are related to the values for $L_{\text {ZAMS }}$ and $L_{\text {TMS }}$ given in the work of Guo et al. (2009) by Eqs. 9 and 12. The corresponding terms in Eq. 17 relate to the outer boundary. Stars with masses less than $0.2 M_{\odot}$ have been excluded as it is unlikely that the mass and metallicity of their circumstellar disks will be high enough to support the formation of planets above the habitable planet mass limit of 0.3 Earth masses $\left(M_{\oplus}\right)$ (Raymond et al., 2007).

The approach outlined above can then be used to provide an approximation of the gradual evolution of the HZ distance. The $\mathrm{HZ}$ transition rate can be used to estimate the $\mathrm{HZ}$ 
FIG. 5. Position of 27 Kepler candidates within the ZAMS habitable zone. The outer dashed lines represent a $100 \%$ cloud cover scenario, the dotted lines 50\%, and the solid lines the "classic" HZ.

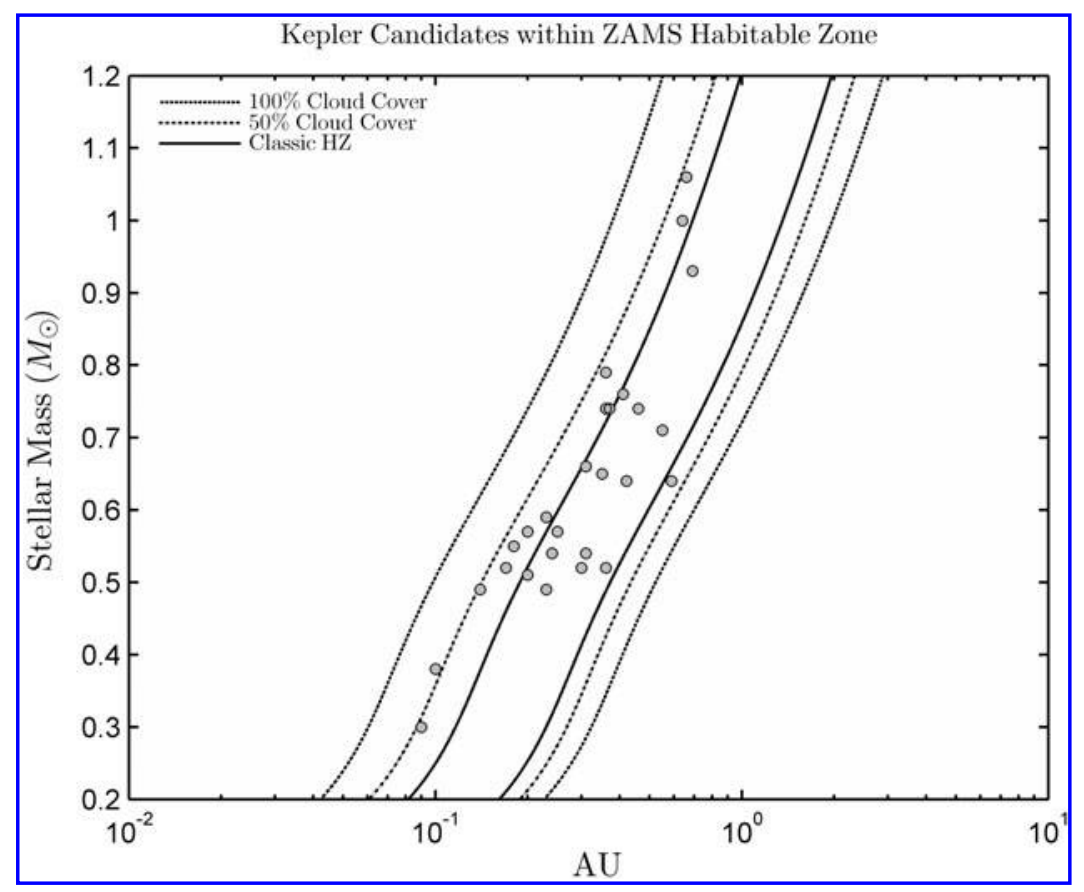

lifetime of a planet, which we consider to terminate at the stage at which the inner boundary of the HZ and the semimajor axis of the planet's orbit become identical, signaling its entry into the hot zone of the star:

$$
\tau_{\mathrm{h}}=\left(\frac{a_{\text {planet }}-\mathrm{HZ}_{\text {inner }}^{\text {ZAMS }}}{\mu_{\text {inner }}}\right)
$$

where $a_{\text {planet }}$ denotes the semimajor axis. Substituting the outer transition rate $\left(\mu_{\text {outer }}\right)$ and $\mathrm{HZ}_{\text {outer }}^{\mathrm{ZAMS}}$ into Eq. 18 , this technique can be used to estimate the transitional period, or prehabitable phase, of a Class II habitable planet within the outer boundary zone, thereby effectively approximating its entry into the HZ area and initiating its HZ lifetime.

The more complex approach to this problem is to derive estimates for the change in the HZ boundaries over time by using the hybrid polynomial/linear relationship between luminosity, mass, and time (Eqs. 3 and 4). This method produces well-constrained $\left(r^{2}>0.99\right)$ second-order polynomial fits between $\mathrm{HZ}$ distance and $\tau$ at $0.1 M_{\odot}$ intervals. As before, attaining a value for the $\mathrm{HZ}$ lifetime $\left(\tau_{\mathrm{h}}\right)$ can then be done by determining the value of $\tau$ when the $\mathrm{HZ}$ distance is identical to the semimajor axis of the planet of interest $\left(a_{\text {planet }}\right)$.

Based on the predictions of other studies, the polynomial fits probably overestimate $\tau_{\mathrm{h}}$, while the linear fits are likely too conservative (Caldeira and Kasting, 1992; Goldblatt and Watson, 2012). The difference in the HZ lifetime that results is due to variations in the slope of the $L(t)$ distribution and the behavior of $L$ in the early and late stages of the star's lifetime. This, however, translates into a significant dissimilarity in the rate of the HZ boundary transition rate and consequently the HZ. We have included results from both schemes where possible to act as upper and lower bounds of the HZ lifetime and also to illustrate the errors and uncertainties associated with these calculations, originating from the strong control of the shape of the $L(\tau)$ slope on the output. More research and better constraints of main sequence lifetime, as well as the change in luminosity over this period, are required to further reduce these uncertainties.

The model is calibrated for Earth; therefore we assume "Earth-like" conditions on the planets included in the test sample. These include Mars (or rather, an Earth-like planet at Mars' orbital distance) as well as seven exoplanets confirmed to be within the HZ of their stars by the online Habitable Exoplanets Catalog maintained by the Planetary Habitability Laboratory at the University of Puerto Rico, Arecibo (Planetary Habitability Laboratory, 2012) as of 11 December 2012. These are Kepler 22b, HD 85512b, HD 40307g, Gliese 163c, Gliese $667 \mathrm{Cc}$, Gliese 581d, and Gliese 581g. We note that Gliese 581g and HD 40307g are considered "planet candidates," and more data are required to definitively classify them as planets. Twenty-seven unconfirmed Kepler candidates, also identified to be orbiting in their stars' $\mathrm{HZ}$ by the Habitable Exoplanets Catalog (Fig. 5), were also included in the results. We also note that the Habitable Exoplanets Catalog utilizes the HZ model of Selsis et al. (2007), which includes the effect of reflective clouds mentioned previously.

\section{Results}

Equations 16 and 17 for the linear transition rate of the inner and outer $\mathrm{HZ}$ boundaries for stars between 0.2 and 1.2 $M_{\odot}$ over their entire main sequence lifetime are displayed in black in Fig. 6 . Inner boundary $\mathrm{HZ}$ transition rates $\left(\mu_{\text {inner }}\right)$ fall in a range between $9.5 \times 10^{-4}$ and $1.06 \times 10^{-1} \mathrm{AU} \mathrm{Gyr}{ }^{-1}$; outer transition rates $\left(\mu_{\text {outer }}\right)$ lie between $2 \times 10^{-3}$ and $2.1 \times 10^{-1} \mathrm{AU} \mathrm{Gyr}{ }^{-1}$, depending on stellar mass. $\mathrm{M}$ type and $\mathrm{K}$ type stars ( $>0.08$ to $0.8 \mathrm{M}_{\odot}$ ) have lower rates of $\mu$ because of their long hydrogen-burning lifetimes relative to the $G$ class Sun, which experiences rates of $\mu_{\text {inner }}$ of approximately $5 \times 10^{-3}$ AU Gyr ${ }^{-1}$ and $\mu_{\text {outer }}$ of $0.1 \times 10^{-1}$ AU Gyr ${ }^{-1}$. The outer HZ boundary exhibits a slightly more rapid rate of outward migration under all mass classes relative to the inner boundary due to the fourth-power proportionality 


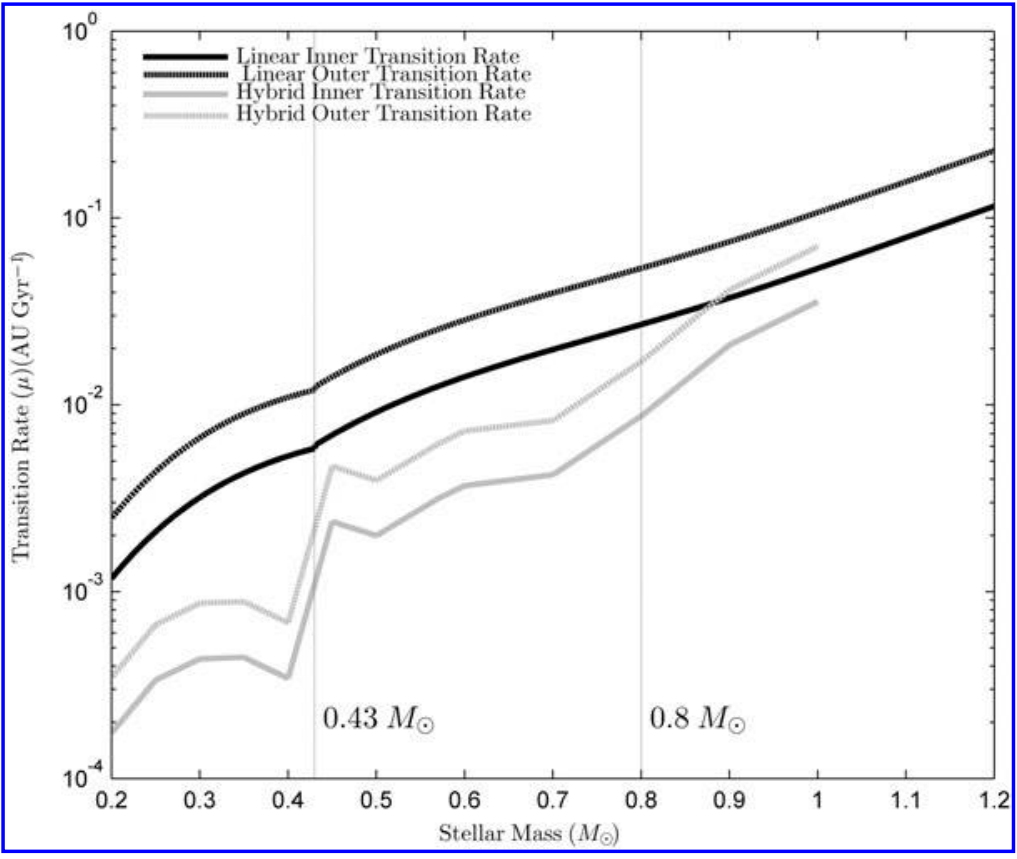

FIG. 6. Radiative HZ boundary transition rates as a function of stellar mass in astronomical units $(\mathrm{AU})$ per $10^{9}$ years $(\mathrm{Gyr})$ when using the linear migration model (black lines) shown in Eqs. 16 and 17, as well as the hybrid luminosity evolution model (gray lines) for the first 12.6 Gyr of stellar evolution. between planetary effective temperature and distance from the star. As stellar main sequence age has a strong control on $\mu$, Eqs. 5, 6, and 7 result in three distinct trends between mass classes: lower-mass $\left(0.2-0.43 M_{\odot}\right)$, mid-range $\left(0.43-0.8 M_{\odot}\right)$, and higher-mass $\left(0.8-1.2 M_{\odot}\right)$ linear transition rates can be calculated with the second-order polynomials provided in Table 4 .

The gray lines shown in Fig. 6 represent HZ transition rates when using the hybrid stellar evolution model described above, and also shown in Fig. 3, for stars between 0.2 and $1 M_{\odot}$ (at $0.1 M_{\odot}$ intervals) between 0.5 and 12.6 Gyr of stellar evolution, as set by the Baraffe et al. (1998) model. This model does not take the full main sequence luminosity evolution of stars older than 12.6 Gyr into account; therefore the results are less well constrained. Lower-mass stars exhibit an order of magnitude slower transition rates under this scheme, with the effect becoming less pronounced with increasing mass.

Substituting these values into Eq. 18, it is possible to estimate the approximate $\mathrm{HZ}$ lifetime of a planet, where its semimajor axis $(a)$ is known. The results are shown in Fig. 7. The two lines, denoted the "Typical Habitable Zone Lifetime," running through this figure are normalization factors produced by calculating the HZ lifetime of a hypothetical planet formed directly in the center of the HZ at the ZAMS stage under each mass class considered and under both the linear and hybrid version of the HZ lifetime model. They are included to illustrate the trend that planets orbiting smaller- mass stars tend to have longer HZ lifetimes, when accounting for their relative location within the HZ. Note that the typical HZ lifetime for the linear $L(t)$ relationship is significantly less than that of the typical HZ lifetime returned when using the hybrid $L(t)$ fits due to lower rates of boundary transition shown in Fig. 6. We have linearly extended the hybrid fit from 1 to $1.2 M_{\odot}$. Individual deviations from this line illustrate a departure from the theoretical "typical habitable zone lifetime" of the star system in time but also in space, as planets that form nearer the inner edge will have relatively shorter $\mathrm{HZ}$ lifetimes, irrespective of the life span of their star. Therefore, planets nearer the outer edge will plot above this line, while those nearer the inner edge and hot zone will plot below. The exception to this is Mars; while not in the $\mathrm{HZ}$ at present, its future $\mathrm{HZ}$ lifetime (assuming Earthlike conditions) will be constrained by the main sequence lifetime of the Sun. The HZ lifetime of planets included in this plot follows the linear (more conservative) version of the model.

Unless stated, the figures provided for the planets and planetary candidates discussed below (and in Fig. 7) refer to the departure of a Class I habitable planet from the classic HZ (Lammer et al., 2009). Where necessary, it is also possible to estimate the timing and duration of the transition from the classic $\mathrm{HZ}$ to the uncertain inner boundary/hot zone, where planets require $50 \%$ or greater cloud cover to maintain habitable surface temperatures. The results of the following section are summarized in Fig. 8.

Table 4. Fitting Equations for Linear Habitable Zone Transition Rates (AU Gyr ${ }^{-1}$ ) as a Function of Star Mass

\begin{tabular}{lll}
\hline $\mathrm{M}_{\odot}$ & \multicolumn{1}{c}{ Inner Boundary Transition $\left(\mu_{\text {inner }}\right)$} & Outer Boundary Transition $\left(\mu_{\text {outer }}\right)$ \\
\hline $0.2-0.43$ & $-0.0108 M_{\odot}^{2}+0.0189 M_{\odot}-0.0025$ & $-0.0262 M_{\odot}^{2}+0.0413 M_{\odot}-0.0053$ \\
$0.43-0.8$ & $0.0716 M_{\odot}^{2}+0.0324 M_{\odot}-0.0046$ & $0.142 M_{\odot}^{2}+0.0642 M_{\odot}-0.0094$ \\
$0.8-1.2$ & $0.4022 M_{\odot}^{2}+0.6084 M_{\odot}-0.2554$ & $0.7877 M_{\odot}^{2}+1.1894 M_{\odot}-0.4995$ \\
\hline
\end{tabular}

All fits: $r^{2}>0.999$. 


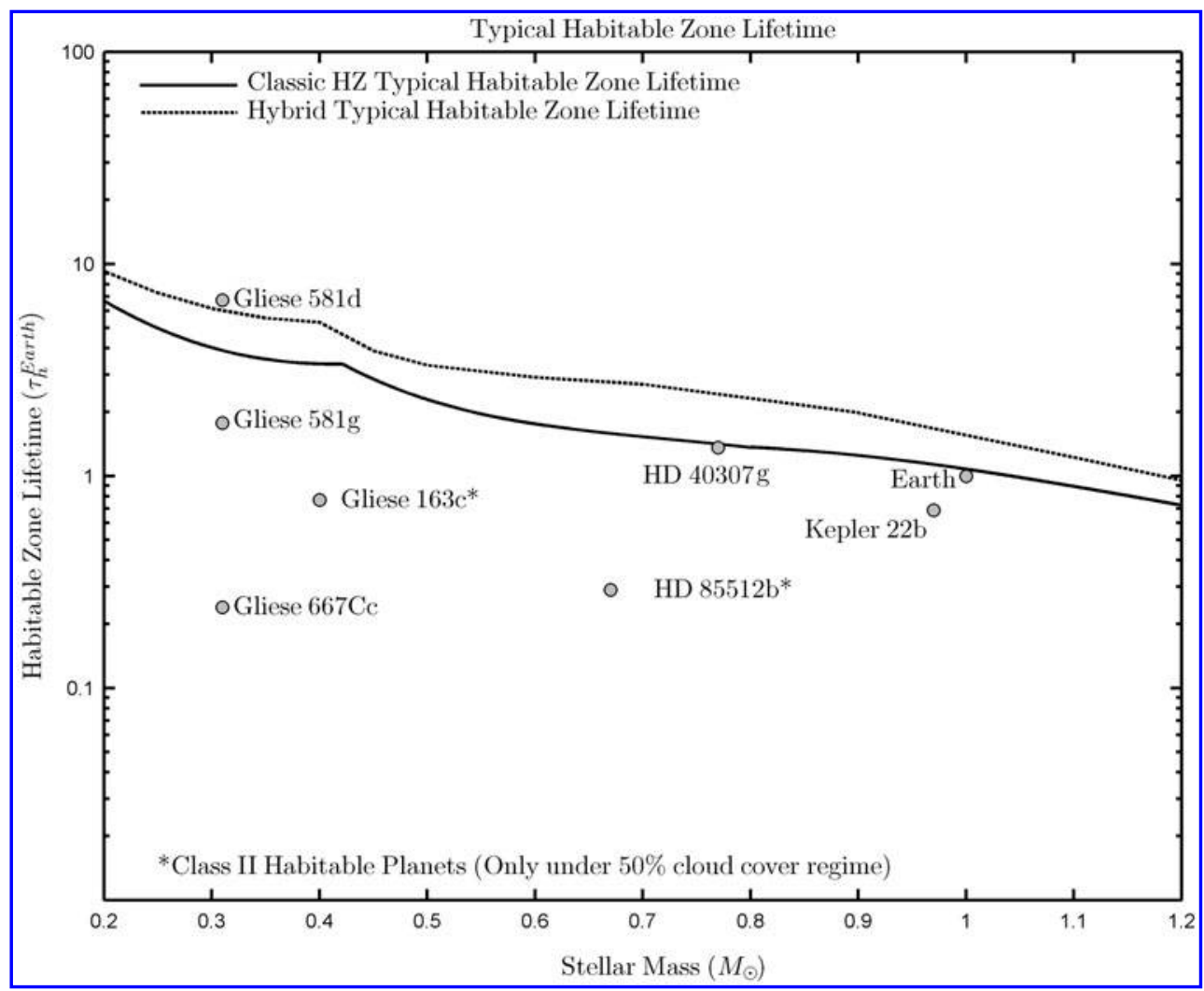

FIG. 7. Habitable zone lifetime $\left(\tau_{\mathrm{h}}\right)$ as a function of stellar mass, where $\tau_{\mathrm{h}}$ is normalized to $\tau_{\mathrm{h}}^{\text {Earth }}$ (6.29 Gyr). The "typical habitable zone lifetime" lines running through the plot represent the HZ lifetime of a hypothetical planet that formed in the very center of the classic HZ at the ZAMS stage of stellar evolution under different stellar luminosity evolution models. Also plotted are Earth and seven HZ exoplanets. All planetary $\tau_{\mathrm{h}}$ are based on the classic $\mathrm{HZ}$ and linear luminosity evolution, with the exception of HD 85512b and Gliese 163c, which are both Class II habitable planets that can only be considered within the HZ under a $50 \%$ cloud cover regime.

The linear $L$ model predicts a total HZ lifetime for Earth $\left(\tau_{\mathrm{h}}^{\text {Earth }}\right)$ of 6.29 Gyr, corresponding to a habitable-to-inhabitable transition occurring approximately 1.75 Gyr from present, taking the age of the planet as 4.54 Gyr. The hybrid $L$ fit returns a $\tau_{\mathrm{h}}^{\text {Earth }}$ of approximately $7.79 \mathrm{Gyr}$, the HZ lifetime terminating in 3.25 Gyr. The 1.5 Gyr difference between these estimates provides an upper and lower limit on the habitable period of Earth. Similarly, Venus' HZ lifetime was on the order of 1.3 Gyr from ZAMS.

It should be noted that our approach in this case is primarily stellar such that we focus on the evolution of main sequence stars rather than the biogeochemical processes that take place on a given planet in its orbit, which allows for application of this model to Earth analog planets with otherwise unknown properties as a first-order assessment of their habitability through time. Therefore, we consider only the position of these planets in space and do not take into account other planetary characteristics that are certain to have bearing on their overall habitability, including planetary mass, composition, tectonic and magnetic activity, or atmospheric and climatic conditions beyond those parameterized in the $\mathrm{HZ}$ model. These assumptions should be considered when interpreting the model results, which are intended to be presented along with other metrics to produce a more robust habitability index.

Estimates provided by other workers (e.g., Caldeira and Kasting, 1992; Goldblatt and Watson, 2012) are on the order of 2-2.5 Gyr from present, due to the use of radiative transfer models of differing complexity and structure optimized for Earth's atmosphere. The results from a novel approach based on modeling the likely evolution of global biological community structure in response to increasing $L$ adopted by O'Malley-James et al. (2012) suggest that unicellular life could persist in refugia on Earth 2.8 Gyr from present. Authors using a geodynamical approach have predicted the collapse of habitable conditions on Earth 1.4 Gyr from present (Franck et al., 2000). However, under a regime of increasing solar luminosity, which is estimated to be $118 \%$ of the present-day value at $1.75 \mathrm{Gyr}$, we consider an irreversible runaway greenhouse scenario to be more likely (Kasting, 1988; Goldblatt and Watson, 2012).

Adopting the outer boundary transition rate of the solar $\mathrm{HZ}$ ( $\left.\mu_{\text {outer }}\right)$ as $0.1 \mathrm{AU} \mathrm{Gyr}^{-1}$, the $\mathrm{HZ}$ for Earth-like planets will extend beyond the orbit of Mars approximately 1.57 (polynomial fit) and 1.66 (linear model) Gyr from present and remain beyond 1.5 AU until the end of Sol's main sequence lifetime $\left(\tau_{\mathrm{Sol}}=10.9 \mathrm{Gyr}\right)$, approximately 4.7 Gyr later (Nebojsa, 2004). Despite its residence in the HZ at this late stage in Sol's main sequence lifetime, it is unlikely that Mars will be "habitable" as it has extremely low atmospheric pressure and limited geodynamics to power global geochemical cycling processes. We ignore the effect that Mars' complex orbital evolution, driven by its secularly increasing orbital eccentricity, may have on its future semimajor axis (Laskar et al., 2004). 

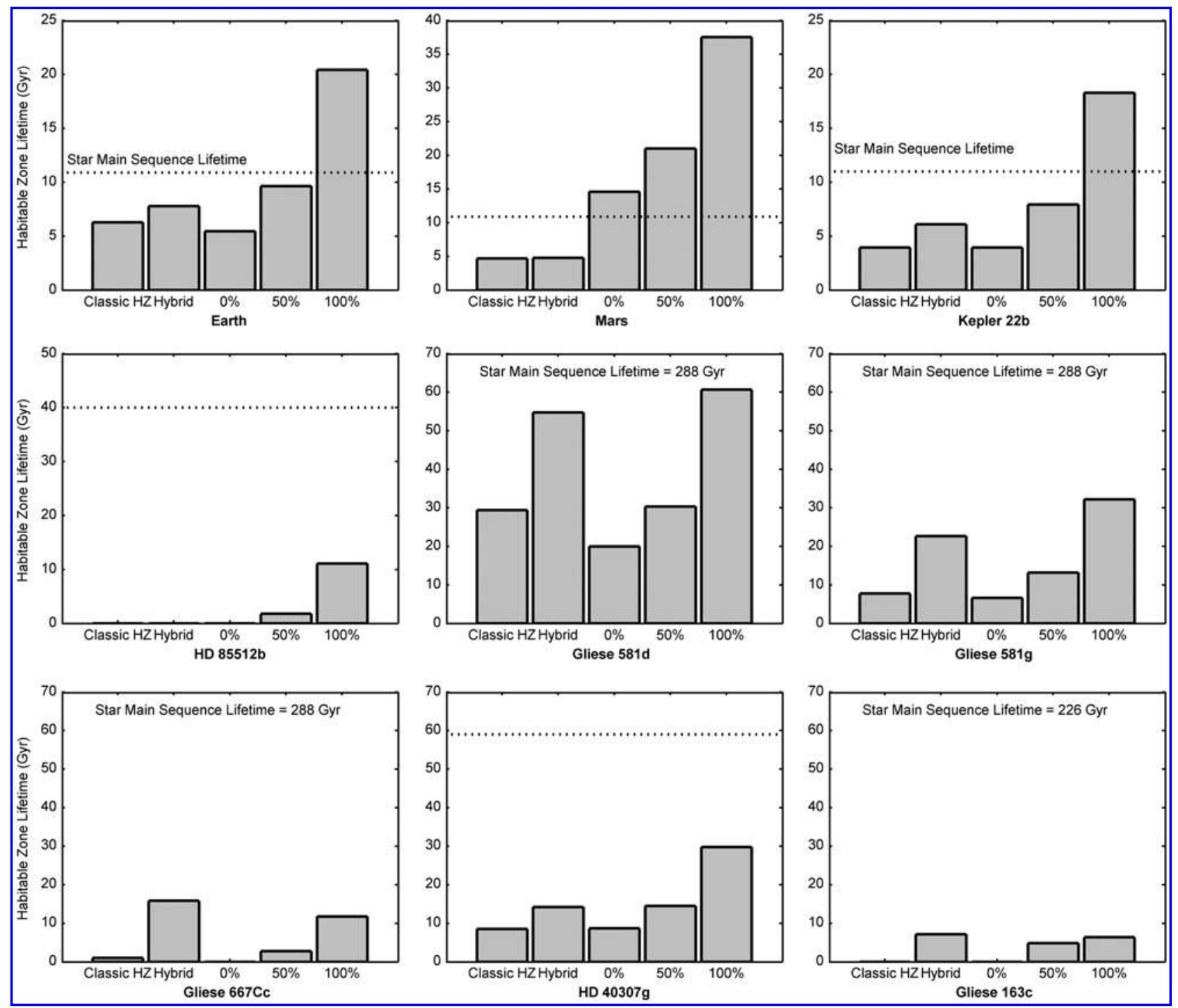

FIG. 8. Habitable zone lifetimes for Earth, Mars, and seven HZ exoplanets using differing HZ models. "Classic HZ" refers to the radiative HZ advanced by Kasting et al. 1993 (Eqs. 9 and 12), "Hybrid" to the relationship outline in the previous section detailing the use of polynomial fits for $L\left(M_{\odot}, \tau\right)$ to $12.6 \mathrm{Gyr} .0 \%, 50 \%$, and $100 \%$ refer to the $0 \%$, $50 \%$, and $100 \%$ cloud cover regimes proposed by Selsis et al. (2007), respectively.

Super-Earth exoplanet Kepler 22b has a slightly more concise $\tau_{\mathrm{h}}$ of $4.31 \mathrm{Gyr}\left(\tau_{\mathrm{h}} / \tau_{\mathrm{b}}^{\text {Earth }}=0.69\right)$ or $6.11 \mathrm{Gyr}$ $\left(\tau_{\mathrm{h}} / \tau_{\mathrm{b}}^{\text {Earth }}=0.79\right)$ when using the hybrid $\tau_{\mathrm{h}}$ version, due to its analogous orbital $(a=0.84 \mathrm{AU})$ and parent star $\left(M_{\odot}=0.97\right)$ characteristics. HD 40307g, a Super-Earth or possibly Neptune-sized exoplanet orbiting a $\mathrm{K}$ class star with a mass of $0.77 \mathrm{M}_{\odot}$, has a relatively extensive $\mathrm{HZ}$ lifetime, estimated to be on the order of $8.6 \mathrm{Gyr}\left(\tau_{\mathrm{h}} / \tau_{\mathrm{b}}^{\text {Earth }}=1.36\right)$ to $14.2 \mathrm{Gyr}$ $\left(\tau_{\mathrm{h}} / \tau_{\mathrm{b}}^{\text {Earth }}=2.25\right)$ (Barnes et al., 2009).

Orbital neighbors Gliese 581d and Gliese $581 \mathrm{~g}$ exhibit markedly differing values of $\tau_{\mathrm{h}}$ despite their shared red dwarf parent star $\left(M_{\odot}=0.31\right)$ because of their disparate orbits. The existence of Gliese $581 \mathrm{~g}$ remains unsettled to date, and more observations are required to definitively confirm the presence of the planet (Forveille et al., 2011; Gregory, 2011; Tuomi, 2011; Vogt et al., 2012). We have included it here as an interesting candidate with which to test the model; the results we present here make no attempt to confirm or validate the existence of the planet itself. While both planets in this system are in the ZAMS habitable zone, which extends from 0.119 to $0.23 \mathrm{AU}$, the innermost super-Earth planet Gliese 581g ( $a=0.14 \mathrm{AU})$, potentially the most habitable exoplanet found to date if confirmed, has a $\tau_{\mathrm{h}}$ of 11.2 Gyr, approximately 5 Gyr longer than that of Earth $\left(\tau_{\mathrm{h}} / \tau_{\mathrm{b}}^{\text {Earth }}=1.78\right)$ (Planetary Habitability Laboratory, 2012). The polynomial $L(\tau)$ fits results in a substantially longer $\mathrm{HZ}$ lifetime of approximately 22.7 Gyr $\left(\tau_{\mathrm{h}} / \tau_{\mathrm{h}}^{\text {Earth }}=2.94\right)$. Because of its position nearer the outer edge of the classic HZ and the long main sequence lifetime of its star, Gliese 581d $(a=0.22$ $\mathrm{AU})$ will remain in the $\mathrm{HZ}$ for $42.4 \mathrm{Gyr}\left(\tau_{\mathrm{h}} / \tau_{\mathrm{h}}^{\text {Earth }}=6.74\right)$ under the linear model and 54.7 Gyr $\left(\tau_{\mathrm{h}} / \tau_{\mathrm{h}}^{\text {Earth }}=7.08\right)$ when using the hybid fit, the longest $\tau_{\mathrm{h}}$ of any exoplanet discovered to date.

Gliese 667Cc, also orbiting a $0.31 M_{\odot}$ red dwarf, has a HZ lifetime of $1.5 \mathrm{Gyr}\left(\tau_{\mathrm{h}} / \tau_{\mathrm{h}}^{\text {Earth }}=0.24\right)$, due to its proximate orbit $(a=0.123)$, straddling the inner boundary of the habitable 


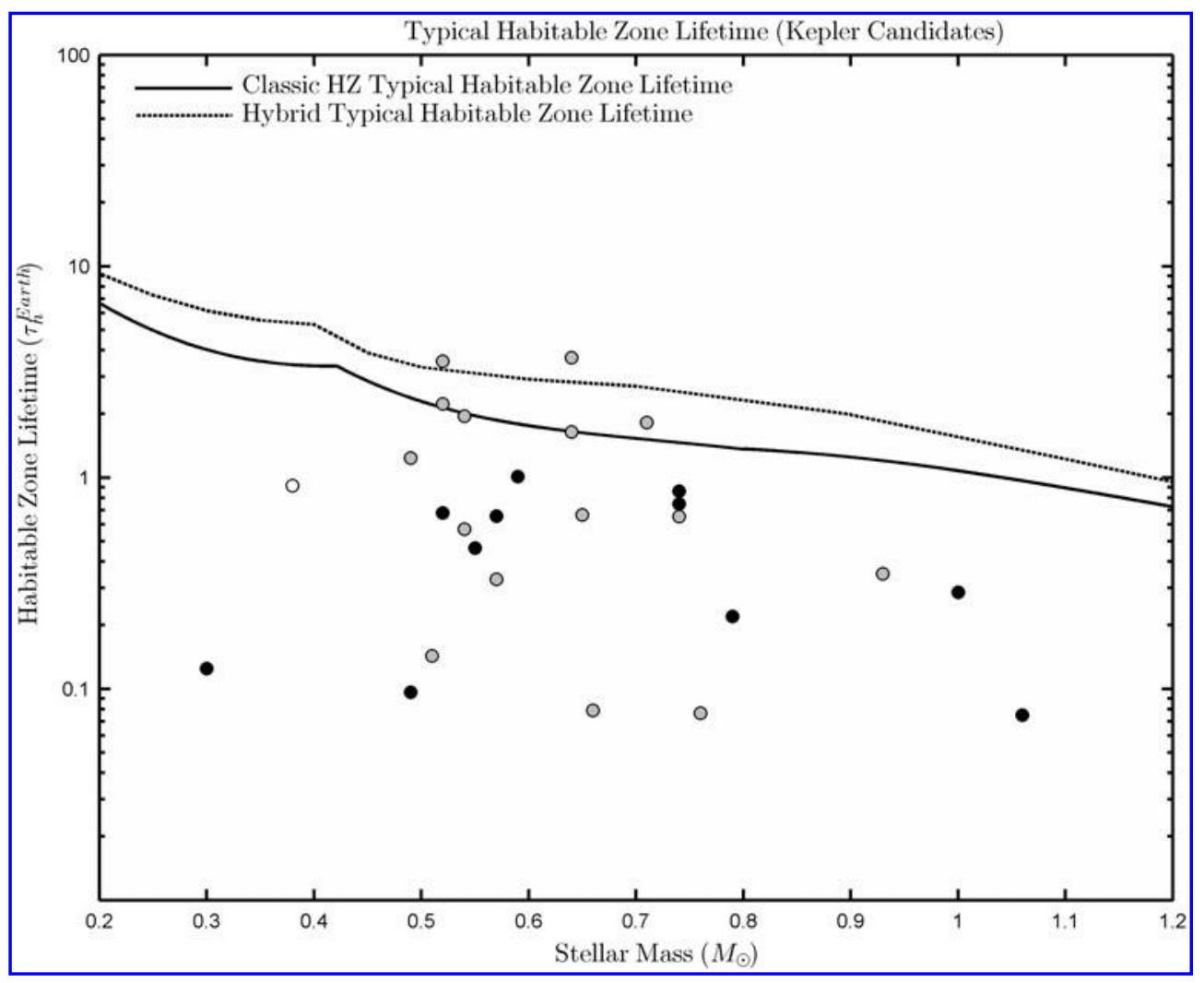

FIG. 9. Habitable zone lifetime $\left(\tau_{\mathrm{h}}\right)$ as a function of stellar mass, where $\tau_{\mathrm{h}}$ is normalized to $\tau_{\mathrm{h}}^{\text {Earth }}$ (6.29 Gyr), for $27 \mathrm{Kepler}$ candidates detected within the HZ of their stars. We have differentiated between Class I planets (gray markers-within the classic HZ) $(n=15)$, Class II planets (solid black markers-within the uncertain inner boundary/hot zone) $(n=11)$ habitable only under a $50 \%$ cloud cover regime, and Class II planets (white marker) habitable only under conditions of $100 \%$ cloud cover $(n=1)$ (see discussion for further commentary).

zone $\left(\mathrm{HZ}_{\text {inner }}^{\mathrm{ZAMS}}=0.119 \mathrm{AU}\right)$. The polynomial $L$ trend predicts a wider $\mathrm{HZ}$ for this star $\left(\mathrm{HZ}_{\text {inner }}\right.$ at $\left.0.5 \mathrm{Gyr}=0.109 \mathrm{AU}\right)$, resulting in a longer HZ lifetime, on the order of 15.9 Gyr. The order-of-magnitude discrepancy between the linear and fitted HZ lifetimes for this planet remains difficult to reconcile; our initial conclusion is that the polynomial model performs poorly when considering very small distances between the semimajor axis of the planet and the inner boundary of the $\mathrm{HZ}$, coupled with a different estimation of the initial $\mathrm{HZ}$ distance to that of the linear model.

HD 85512b $\left(a=0.26\right.$ AU, $\left.M_{\odot}=0.67\right)$ and Gliese $163 \mathrm{c}$ $\left(a=0.125 \mathrm{AU}, M_{\odot}=0.4\right)$ represent complex cases and illustrate some of the uncertainty and weaknesses in our understanding of the complexities of the HZ. Orbiting near the highly ambiguous inner boundary/hot zone transition at ZAMS, these planets can only be considered to be within the $\mathrm{HZ}$ under a $50 \%$ cloud cover scenario (see Eqs. 8 and 9), and accordingly we would argue that their residence time within the HZ is uncertain (Kaltenegger et al., 2011). Following the work of Lammer et al. (2009), we designate HD 85512b and Gliese 163 c as Class II habitable planets (within the inner or outer edge of the HZ). Nevertheless, under the assumption of reflective cloud cover of at least $50 \%$, the effective planetary temperature on HD 85512b and Gliese 163c may be within the bounds of habitability for $1.8 \mathrm{Gyr}\left(\tau_{\mathrm{h}} / \tau_{\mathrm{h}}^{\text {Earth }}=0.29\right)$ and 4.8 Gyr $\left(\tau_{\mathrm{h}} / \tau_{\mathrm{h}}^{\text {Earth }}=0.77\right)$ from ZAMS, respectively. These planets remain out of bounds of the hybrid polynomial $L$ model, and HD $85512 \mathrm{~b}$ will be subject to further discussion in the following section.

We also chose to include 27 Kepler candidates in our sample to illustrate the ability for the model to compute HZ lifetimes for larger data sets and use candidates that display a broad range of host star masses $\left(0.3-1.06 M_{\odot}\right)$ and orbital distances. We differentiated between Class I candidates $(n=15)$ in the more robust "classic" HZ (i.e., <50\% cloud cover) and Class II planets only considered to be habitable under a $50 \%$ and $100 \%$ cloud cover regime (Lammer et al., 2009). Figure 5 illustrates the distribution of candidate planets throughout the HZ, 12 of which fall within the uncertain hot zone/inner boundary transition zone. The theoretically habitable zone lifetimes of these candidate planets fall within 0.47 and $23.2 \mathrm{Gyr}$, as shown in Fig. 9.

\subsection{Are these planets still in the habitable zone?}

Arguably, the most useful practical application of this model is for ascertaining the absolute remaining $\mathrm{HZ}$ lifetime of an individual exoplanet when the age of its host star has been determined. For many of the planets contained in our sample, however, the ages of their stars are not well known. For example, the age of star Kepler 22 remains unknown to date, Gliese 667C has an age <2 Gyr, and the age of Gliese 163 remains very poorly constrained (Planetary Habitability Laboratory, 2012). 
The age of Gliese 581 is estimated as $8 \pm 1$ Gyr, suggesting that both planet candidates remain with the $\mathrm{HZ}$ even under the more conservative classic HZ conditions (Selsis et al, 2007). Similarly, star HD 40307 has an estimated age of $\sim 4.5$ Gyr, suggesting that the planet HD $40307 \mathrm{~g}$ is still within the $\mathrm{HZ}$ of its host star and will remain there for a further $4.1 \mathrm{Gyr}$ under the classic HZ scenario (Tuomi et al., 2013). Having orbited within the $\mathrm{HZ}$ of its host star $\left(0.77 \mathrm{M}_{\odot}\right)$ for a comparable time to that of Earth, we suggest that this planet candidate makes for a very interesting subject for further study.

The age of HD 85512 is given as $5.61 \pm 0.61$ Gyr; however, some uncertainty also exists between the spectroscopic and isochrone data for this star resulting in a mass estimate of $0.69 \pm 0.08 M_{\odot}$ (Pepe et al., 2011). Other workers also initially assessed the habitability of the planet HD 85512b, using the Selsis et al. (2007) model, and returned a similar result to that presented above, placing the planet in the $50 \%$ cloud cover zone (Kaltenegger et al., 2011). However, it does not appear that they have considered the evolution of the luminosity of this star over its main sequence lifetime to date. Even given the mass and age range, and assuming an Earth analog atmosphere, our results suggest that HD $85512 \mathrm{~b}$ is no longer in the HZ (except under 100\% cloud cover) and has not been for several billion years. While the estimated semimajor axis of HD $85512 b$ is $0.26 \mathrm{AU}$, the inner boundary of the HZ for a $0.65 M_{\odot}$ star (lower estimate) with an age range between 5 and 6.22 Gyr is $\sim 0.36$ and $\sim 0.38$ AU, respectively, under the classic HZ scenario and $0.28-0.30$ AU under $50 \%$ cloud cover regime. For the upper mass limit $\left(0.73 \mathrm{M}_{\odot}\right)$, the classic HZ inner limit is $\sim 0.47 \mathrm{AU}$ at $5 \mathrm{Gyr}$ and $\sim 0.48 \mathrm{AU}$ at $6.22 \mathrm{Gyr}$, while the $50 \%$ cloud cover scenario returns limits of $\sim 0.32$ and $\sim 0.33 \mathrm{AU}$, respectively.
The current measured luminosity of HD 85512 is $0.126 \pm 0.008 L_{\odot}$ (Pepe et al., 2011). This value agrees well with our modeled luminosity $\left(L_{\odot}=0.1256\right)$ for a star of this age (5.61 Gyr) if its mass is $\sim 0.67 \mathrm{M}_{\odot}$. With these parameters, the HZ inner limit is $0.385 \mathrm{AU}$ when using the classic model and $0.30 \mathrm{AU}$ under the $50 \%$ cloud cover scenario. The classic and 50\% cloud cover HZ lifetimes are 0 and 1.8 Gyr, respectively. Accordingly, we would suggest that HD 85512b should no longer be considered a viable habitable planet candidate.

\subsection{Continuously habitable zones}

The linear model also allows for the production of plots of "continuously habitable zones" (CHZs) (Fig. 10). These describe an area around a star that would remain within the radiative $\mathrm{HZ}$ for a given time, first hypothesized by Hart (1979) and developed further by Kasting et al. (1993). For example, Fig. 10 illustrates the CHZs for 1, 5, 10, and 50 Gyr. Our 5 Gyr CHZ is comparable to that of Kasting et al. (1993), extends across all mass classes considered, and is especially pronounced around solar-mass stars (0.94-1.45 AU) and above. The 10 Gyr CHZ is also fairly encompassing across the stellar mass range considered, but stars above 1.03 $M_{\odot}$ are unlikely to have main sequence lifetimes in excess of 10 Gyr. Solar mass stars have a 10 Gyr CHZ extending from approximately 1.18-1.45 AU, excluding both Earth and Mars, as expected. Only low-mass stars (between 0.2 and roughly $0.31 M_{\odot}$ ) have main sequence lifetimes that are able to support a CHZ of $50 \mathrm{Gyr}$, as shown in the bottom right plot of Fig. 10. We suggest that Earth analog exoplanets or exomoons found to be orbiting within these areas should be identified as particularly interesting candidates for continued observation and study, especially if coupled with a

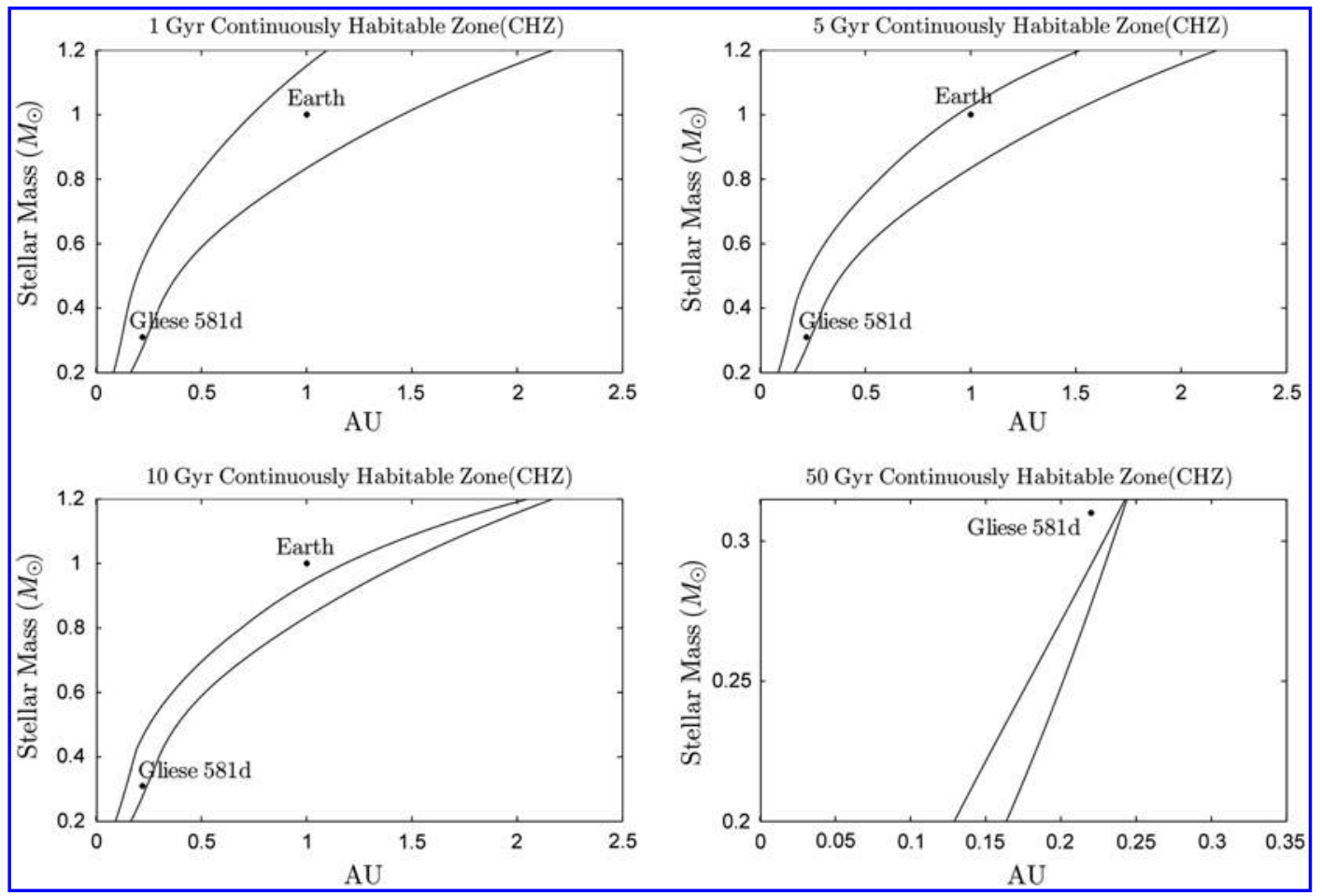

FIG. 10. 1, 5, 10, and 50 Gyr CHZs, using the linear model as described above. Earth and Gliese 581d are included for reference. Note the change of scale of both axes in the $50 \mathrm{Gyr} \mathrm{CHZ}$ plot, bottom right. 
FIG. 11. Main sequence age as a function of stellar mass for stars between 0.2 and 1.2 $M_{\odot}$ as well as the ratio of the typical $\mathrm{HZ}$ lifetime $\left(\tau_{\mathrm{h}}\right)$ to that of $\tau$.

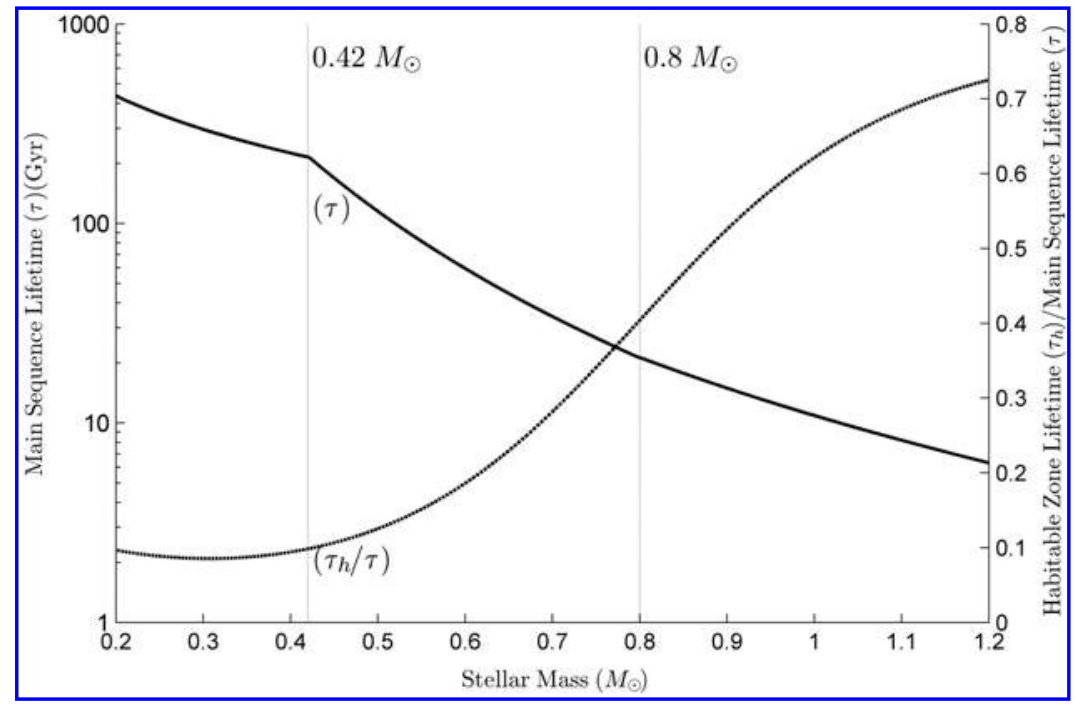

good estimate for the relative age of their star, as they may have spent an extensive zone lifetime of time within the HZ.

Applying the linear HZ lifetime $\left(\tau_{\mathrm{h}}\right)$ model and modeled main sequence lifetime $(\tau)$, it is possible to devise a simple ratio between these relationships, shown in Fig. 11 and Table 5. Lower-mass stars, while exhibiting extremely long main sequence lifetimes, have proportionally lower $\tau_{\mathrm{h}} / \tau$ ratios than higher-mass stars. For example, despite a main sequence lifetime of $436 \mathrm{Gyr}$ and typical HZ lifetime of 42 Gyr, a $0.2 M_{\odot}$ star has a $\tau_{\mathrm{h}} / \tau$ ratio of 0.09 , compared to a solar mass star with a $\tau$ value of $10.9 \mathrm{Gyr}$ and a $\tau_{\mathrm{h}}$ of 6.77 Gyr $-\mathrm{a} \tau_{\mathrm{h}} / \tau$ ratio of 0.62 . Therefore, a planet that forms in the center of a solar-mass star's HZ will be in the HZ for $62 \%$ of the duration of its host star's main sequence lifetime, compared to only $9-10 \%$ for a planet in the orbit of a $0.2 M_{\odot}$ star. This relationship is both a result of the lower rates of stellar luminosity evolution resulting in proportionally lower rates of $\mathrm{HZ}$ boundary transition rates and the fact that the difference (in space) between the inner and outer positions of the HZ at ZAMS and TMS is relatively greater around lowermass stars; the HZ "migrates" proportionally farther, covering a greater area.

Table 5. Typical Habitable Zone Lifetime and the Ratio of the Typical Habitable Zone LifeTime $\left(\tau_{\mathrm{h}}\right)$ To That of $\tau$ As a Function of Stellar Mass

\begin{tabular}{lccc}
\hline & $\begin{array}{c}\text { Main } \\
\text { Sequence }\end{array}$ & $\begin{array}{c}\text { Typical } \\
\text { Habitable Zone }\end{array}$ & \\
Mass $\left(\mathrm{M}_{\odot}\right)$ & Lifetime $(G y r)(\tau)$ & Lifetime $(G y r)\left(\tau_{h}\right)$ & $\tau_{h} / \tau$ \\
\hline 0.2 & 436.3 & 42.16 & 0.097 \\
0.3 & 296.6 & 25.34 & 0.085 \\
0.4 & 225.6 & 21.24 & 0.094 \\
0.5 & 115.1 & 14.42 & 0.125 \\
0.6 & 59.5 & 11.07 & 0.186 \\
0.7 & 34.2 & 9.63 & 0.282 \\
0.8 & 21.2 & 8.56 & 0.404 \\
0.9 & 14.9 & 7.86 & 0.526 \\
1 & 10.9 & 6.77 & 0.621 \\
1.1 & 8.2 & 5.62 & 0.686 \\
1.2 & 6.3 & 4.57 & 0.725 \\
\hline
\end{tabular}

\section{Discussion}

We have presented a simple model, synthesizing current research in the area of radiative HZs around main sequence stars to produce some tentative estimates for the theoretically habitable zone lifetimes of exoplanets within these zones. We suggest that the HZ lifetime metric should be incorporated into a broader habitability index for exoplanets.

Our model provides an estimate for the total HZ lifetime $\left(\tau_{\mathrm{h}}\right)$ for Earth of between 6.29 and $7.79 \mathrm{Gyr}$, ending between 1.75 and 3.25 Gyr from present. The implication is that we are roughly $70 \%$ of the way through the HZ lifetime of our planet-the period that Earth will spend within the HZ of the Sun. We can begin to contrast and compare this value with that of other planets, if the age of their star is known, but also use it as a means for quantifying the evolutionary history of Earth.

A reliable estimate of the age of a planet's host star is integral to our understanding of its habitability. Planets Gliese 581d and 581g, as well as HD 40307g, all provide interesting candidates for further observation because estimates of their HZ lifetimes can be used in conjunction with relatively well-known star ages to produce a "snapshot" of their HZ configuration at this point in time. In addition to this, exoplanets like HD 85512b can be effectively excluded from further habitable exoplanet studies as knowledge of the age of its host star, coupled with an estimate of the transition rates of the HZ, reveals a planet now well outside the HZ.

The estimates returned by the model suggest that planets that have formed near the outer edge of the HZ would be in a more conducive orbital configuration for long residence times within the HZ. Planets nearer the inner hot zone boundary will spend less time in the $\mathrm{HZ}$ and may therefore be less suitable for the long-term evolution of complex life. However, the environmental conditions of planets near the cold outer boundaries and hot inner boundaries of their stars' HZs may present further limiting factors for life beyond those considered here.

The boundaries of the HZ, integral to this and many other measures of habitability, are not well constrained. Initial research by Kasting et al. (1993) set boundary conditions that remain in use today. Development of the concept by Selsis et al. (2007) attempted to quantify the effect of clouds in 
terms of planetary habitability, but their parameterization as albedo features should have some bearing on the interpretation of the results of this model. Habitable zone lifetime estimations for some planets under $100 \%$ cloud cover regimes are an order of magnitude greater than the age of the Universe ( $>100 \mathrm{Gyr}$ ). We agree with the conclusions of Goldblatt and Zahnle (2011) and consider the case under which exoplanets remain habitable only under these conditions to be stretching the interpretation of habitability and the $\mathrm{HZ}$ beyond realistically resolvable boundaries. We therefore urge caution in adopting this approach for identifying potentially habitable exoplanets in the future without first addressing this uncertainty by designating them as Class II habitable planets. The planetary processes that control long-term habitability on this scale are not well understood, and it remains beyond the scope of this project to speculate on the temporal dynamics of these cycles.

Parameterizations of complex climate processes will be required to resolve their effects on habitability metrics, and these are not the focus of the present study. From recent climate studies, other workers confirmed a small heating effect from $\mathrm{CO}_{2}$ clouds on Earth-like exoplanets (Hu and Ding, 2010; Wordsworth et al., 2010; Zsom et al., 2012). When considering vertical atmospheric stratification, Kitzmann et al. (2010) reported a maximum $15 \%$ decrease in the $\mathrm{HZ}$ distance due to the albedo effect of low-altitude water clouds, coupled with a $35 \%$ increase in distance attributed to high-level ice clouds.

Habitable environments may exist on planets and moons beyond the HZ (Heller and Barnes, 2013). For example, the ice-covered ocean of Jupiter's moon Europa may harbor the highest astrobiological potential in the Solar System, despite falling 3.85 AU outside the Sun's HZ in the cold depths of the outer Solar System (Pappalardo, 2010). It should be noted that the classic radiative $\mathrm{HZ}$ was initially established as an "astronomical observable" $\mathrm{HZ}$ and represented a great advance in our understanding of the processes that control planetary climate. However, the rigidity of its definitionrequiring liquid water on the surface of a planet-limits the possibility of including otherwise "habitable" subterranean extraterrestrial environments on moons and planets in the cold zone of stars.

As more radiation is absorbed by greenhouse gases and Rayleigh scattering is more efficient when the host star radiates at shorter wavelengths, spectral type also has some control over the boundaries of the HZ (Pierrehumbert, 2010). For example, the HZs of low-mass $M$ dwarfs have been hypothesized to extend further due to a dampening of the ice-albedo feedback mechanism around these stars (Joshi and Haberle, 2012). Observations from the red dwarfs Gliese 436 and GJ 1214 show that they emit much of their radiation in the red and near-infrared portion of the electromagnetic spectrum, at wavelengths greater than $0.7 \mu \mathrm{m}$, and significantly more in the $3-10 \mu \mathrm{m}$ region than would be expected from a "blackbody" hypothesized $M$ type of a similar temperature. The albedo of ice and snow begins to decrease at wavelengths greater than $1 \mu \mathrm{m}$; therefore the albedo of snowand ice-covered surfaces on planets in the orbit of red dwarfs would be proportionally lower than that of the same surface on Earth (or any other planet in orbit around a $\mathrm{G}$ type or $\mathrm{K}$ type star). This results in a weakening of the ice-albedo feedback mechanism and an extension of the $\mathrm{HZ}$ of between $10 \%$ and $30 \%$ (Joshi and Haberle, 2012).
Interpreting the results that have been returned by this simple model should be done with caution. While we have sought to estimate a theoretically habitable zone lifetime, we do not claim that the time that a planet spends within the HZ is the only control over habitability. Several fundamental biogeochemical feedback loops, essentially disconnected from the stellar HZ, operate on present Earth that boost its habitability for multicellular aerobic organisms. The most notable of these is the secular oxygenation of the atmosphere that began with the Great Oxidation Event, approximately 2.4 Gyr ago, that facilitated the evolution of complex eukaryotic organisms (including humans) that exploit energyefficient aerobic respiration (Catling et al., 2005; Holland, 2006). The actual HZ lifetime of any given planet is unlikely to be controlled solely by planetary surface temperatures, and individual worlds may experience a variety of divergent (bio)geochemical evolutionary histories, possibly resulting in markedly different planetary environments to that of Earth. Habitability through time is likely to be as complex and multifaceted as habitability in space.

\subsection{Implications for the anthropic model}

Attempting to quantify the relative timing of biogenesis and the likely duration of the biosphere of habitable planets-from simple life to intelligent observers-has been a key area of research in astrobiology, extending back to the Drake equation first devised in 1961, and the concept remains extremely pertinent to SETI campaigns.

The probabilistic anthropic model of Watson (2008), which draws on the theory of critical evolutionary transitions advanced by Szathmáry and Maynard-Smith (1995) and the "critical steps" model of Carter (1983), proposes that complex biology becomes increasingly probable with longer HZ lifetimes. A small $(n<10)$ number of very difficult evolutionary transitions are needed to be overcome in order for intelligence of space-faring caliber to emerge. On Earth, for example, this stepwise progression began with the origin of life, continued through the transition from replicating molecules to RNA and then DNA, from prokaryotes to eukaryotes and cell differentiation, and concluded with the final step from primate to human societies. This hypothesis may at first appear to suggest an inference of determinism toward intelligence - a pinnacle toward which all evolution aspires. This tendency to view evolution as a progression toward increasing biological complexity and intelligence likely stems from an anthropocentric bias. However, it remains unnecessary to invoke any form of directionality in evolution; the area of focus of SETI campaigns is that of intelligence, which by its very nature is necessarily complex. Similarly, an organism able to alter its planetary environment to the extent at which biosignatures could be detected across interstellar space will undoubtedly require some level of complexity beyond that of simple replicating molecules. The critical steps model is an instrument used to estimate the probability of unlikely evolutionary events, and its applicability is not necessarily limited to considering the emergence of intelligence.

Our results seem to broadly support the conclusions of Watson (2008). Providing an upper limit for the potential life span of the biosphere, we note that critical evolutionary transitions appear to be few in number and approximately 
Table 6. Critical Evolutionary Transitions throughout Earth History AND Their CoRresponding $\tau_{\mathrm{h}}$ VAlues (WATson, 2008)

\begin{tabular}{lll}
\hline$\tau_{h}^{\text {Earth }}$ & Years since formation of Earth & \multicolumn{1}{c}{ Evolutionary transitions on Earth $^{\mathrm{a}}$} \\
\hline$\leq 0.15$ & c. $1 \mathrm{Gyr}$ & $\begin{array}{l}\text { Replicating molecules } \\
\text { RNA to DNA } \\
\text { Prokaryotes to eukaryotes } \\
>0.25 \leq 0.40\end{array}$ \\
& c. $1.5-2.5 \mathrm{Gyr}$ & Asexual clones \\
$>0.55 \leq 0.65$ & c. $3.5-4 \mathrm{Gyr}$ & Cell differentiation \\
$\geq 0.72(14)$ & $4.54 \mathrm{Gyr}$ & Primate to human societies $($ Homo sapiens) \\
$0.72(15)$ & $4.54 \mathrm{Gyr}$ & Current habitable fraction index for Earth \\
1 & $6.29 \mathrm{Gyr}$ & The Earth becomes uninhabitable \\
$\mathbf{0 . 2 8}$ & $\mathbf{1 . 7 5} \mathrm{Gyr}$ & Remaining life span of biosphere \\
\hline
\end{tabular}

${ }^{\mathrm{a}}$ Szathmáry and Maynard-Smith (1995); ${ }^{\mathrm{b}} \mathrm{McDougall}$ et al (2005).

evenly spaced throughout the HZ lifetime of Earth. We would cautiously propose that, assuming that evolution by natural selection is a universal process for biological speciation, the duration of a planet's HZ lifetime may be an important factor limiting the possible complexity that life can evolve on its surface.

Following this approach, planets with a $\tau_{\mathrm{h}} / \tau_{\mathrm{h}}^{\text {Earth }}$ ratio less than 0.7 or 0.8 would be less likely to harbor complex life, but the possibility of prokaryote-like organisms should perhaps not be dismissed even on planets with values as low as 0.2. Following the formation of Earth, it is thought that prokaryote life needed less than a billion years to establish, which appears to be more rapid than expected given the probabilities of the initial critical steps (Altermann and Kazmierczak, 2003; Watson, 2008; Spiegel and Turner, 2012). Lineweaver and Davis (2002) quantified the probability of biogenesis on terrestrial habitable planets older than $1 \mathrm{Gyr}\left(\tau_{\mathrm{h}} / \tau_{\mathrm{h}}^{\text {Earth }}=0.15\right)$ as $>13 \%$ at the $95 \%$ confidence level, drawing on the percieved rapidity of biogenesis on Earth as key, and inferred from this that life is common in the Universe. However, until we have the opportunity to expand our data set of inhabited planets, and refine the probabilities involved in these calculations, these first estimations remain extremely tentative. Table 6 below outlines some of the critical evolutionary transitions that have taken place on Earth and their corresponding $\tau_{\mathrm{h}}$ value (based on the linear version of the HZ boundary transition).

Planets with very low $\tau_{\mathrm{h}}$ ratios, Gliese 667Cc (0.24) for example, would under this classification remain targets of lower astrobiological potential, despite their residence within the HZ. Better constraints on star age would provide further information regarding the relative timing of HZ lifetimes, which could be used in conjunction with their absolute duration, and would avoid the inclusion of planets that are no longer in the HZ, such as HD 85512b. High-value targets for SETI campaigns would be those planets that have been within the $\mathrm{HZ}$ for an equivalent amount of time as Earth, or longer: HD $40307 \mathrm{~g}$, for example.

If the HZ lifetime is the only factor that controls the emergence of life and evolution of complexity required for intelligence, it follows that planets orbiting low-mass stars with long $\mathrm{HZ}$ lifetimes are much more likely to host life and intelligent observers than planets orbiting higher-mass stars. Our results show that low-mass stars have CHZs possibly extending up to $50 \mathrm{Gyr}$, suggesting that any planets orbiting these stars are $\sim 5$ times "more habitable" than planets orbiting more massive stars with lower HZ lifetimes. Previous workers have attempted to build on the original models and quantify this anthropic selection effect for exoplanets, using probabilistic methods. While our estimates for the duration of the HZ lifetimes of M dwarf exoplanets are an order of magnitude less than those discussed by Waltham (2011), we agree with the author's conclusion that the apparent habitability of these planets must be considerably reduced by other environmental factors.

\subsection{Conclusions}

It remains too early to know whether Earth's HZ lifetime is typical of inhabited planets, but from the limited sample provided above it is obvious that planets within the HZ exhibit a large range of $\mathrm{HZ}$ lifetimes, from significantly less than that of Earth to over five times Earth's HZ lifetime. Highlighting the example of HD 85512b, it is imperative that the transitory nature of the boundaries of the HZ be taken into account when considering the habitability of extrasolar planets. These first estimations, which we hope will be refined in the future as our understanding of the evolution of planetary habitability over time improves, provide an interesting first look at yet another fascinating component of planetary habitability.

\section{Acknowledgments}

Andrew Rushby would like to thank Abel Méndez and José Candelaria (and collaborators) at the Planetary Habitability Laboratory for compiling and maintaining the Habitable Exoplanet Catalog. Also, we extend our thanks to John Bochanski for helpful discussions regarding stellar evolution, and two anonymous reviewers for providing useful and constructive comments that significantly improved the manuscript. A.R. also acknowledges the support of a Dean's Scholarship at the University of East Anglia. In addition, Andrew would like to thank his family and Hannah Eldridge for their many years of love and support.

\section{Abbreviations}

$\mathrm{CHZ}$, continuously habitable zone; HZ, habitable zone; TMS, termination of the main sequence; ZAMS, zero-age main sequence. 


\section{References}

Abbot, D.S. and Switzer, E.R. (2011) The Steppenwolf: a proposal for a habitable planet in interstellar space. Astrophys I 735:L27.

Abe, Y., Abe-Ouchi, A., Sleep, N.H., and Zahnle, K.J. (2011) Habitable zone limits for dry planets. Astrobiology 11:443460 .

Altermann, W. and Kazmierczak, J. (2003) Archean microfossils: a reappraisal of early life on Earth. Res Microbiol 154:611-617.

Baraffe, I., Chabrier, G., Allard, F., and Hauschildt, P.H. (1998) Evolutionary models for solar metallicity low-mass stars: mass magnitude relationships and color-magnitude diagrams. Astron Astrophys 337:403-412.

Barnes, R., Jackson, B., Raymond, S.N., West, A.A., and Greenberg, R. (2009) The HD 40307 planetary system: super-Earths or mini-Neptunes? Astrophys I 695:1006-1011.

Barnes, R., Mullins, K., Goldblatt, C., Meadows, V.S., Kasting, J.F., and Heller, R. (2013) Tidal Venuses: triggering a climate catastrophe via tidal heating. Astrobiology 13:225-250.

Caldeira, K. and Kasting, J.F. (1992) The life span of the biosphere revisited. Nature 360:721-723.

Carter, B. (1983) The anthropic principal and its implications for biological evolution. Philos Transact A Math Phys Eng Sci 310:347-363.

Catling, D.C., Glein, C.R., Zahnle, K.J., and McKay, C.P. (2005) Why $\mathrm{O}_{2}$ is required by complex life on habitable planets and the concept of planetary "oxygenation time." Astrobiology 5:415-438.

Claire, M.W., Sheets, J., Cohen, M., Ribas, I., Meadows, V.S., and Catling, D.C. (2012) The evolution of solar flux from $0.1 \mathrm{~nm}$ to $160 \mu \mathrm{m}$ : quantitative estimates for planetary studies. Astrophys J 757, doi:10.1088/0004-637X/757/1/95.

Colaprete, A. and Toon, O.B. (2003) Carbon dioxide clouds in an early dense martian atmosphere. J Geophys Res 108, doi:10.1029/ 2002JE001967.

Des Marais, D.J., Nuth, J.A., III, Allamandola, L.J., Boss, A.P., Farmer, J.D., Hoeler, T.M., Jakosky, B.M., Meadows, V.S., Pohorille, A., Runnegar, B., and Spormann, A.M. (2008) The NASA Astrobiology Roadmap. Astrobiology 8:715-730.

Dotter, A., Chaboyer, B., Jevremović, D., Kostov, V., Baron, E., and Ferguson, J.W. (2008) The Dartmouth Stellar Evolution Database. Astrophys J 178:89-101.

Forveille, T., Bonfils, X., Delfosse, X., Alonso, R., Udry, S., Bouchy, F., Gillon, M., Lovis, C., Neves, V., Mayor, M., Pepe, F., Queloz, D., Santos, N.C., Ségransan, D., Akmenara, J.-M., Deeg, H.J., and Rabus, M. (2011) The HARPS search for southern extra-solar planets XXXII. Only 4 planets in the G1581 system. arXiv:1109.2505.

Franck, S., Block, A., von Bloh, W., Bounama, C., Schellnhuber, H.J., and Svirezhev, Y. (2000) Reduction of biosphere life span as a consequence of geodynamics. Tellus B Chem Phys Meteorol 52:94-107.

Goldblatt, C. and Watson, A.J. (2012) The runaway greenhouse: implications for future climate change, geoengineering and planetary atmospheres. Philos Transact A Math Phys Eng Sci 370:4170-4216.

Goldblatt, C. and Zahnle, K.J. (2011) Clouds and the faint young Sun paradox. Climate of the Past 7:203-220.

Gregory, P.C. (2011) Bayesian re-analysis of the Gliese 581 exoplanet system. Mon Not $R$ Astron Soc 415:2523-2545.

Guo, J., Zhang, F., Chen, X., and Han, Z. (2009) Probability distribution of terrestrial planets in habitable zones around host stars. Astrophys Space Sci 323:367-373.
Hart, M.H. (1979) Habitable zones about main sequence stars. Icarus 37:351-357.

Heller, R. and Barnes, R. (2013) Exomoon habitability constrained by illumination and tidal heating. Astrobiology 13: $18-46$.

Heller, R., Leconte, J., and Barnes, R. (2011) Tidal obliquity evolution of potentially habitable planets. Astron Astrophys 528:A27.

Holland, H.H. (2006) The oxygenation of the atmosphere and oceans. Philos Trans R Soc Lond B Biol Sci 381:903-915.

$\mathrm{Hu}$, Y. and Ding, F. (2010) Radiative constraints on the habitability of exoplanets Gliese 581c and Gliese 581d. Astron Astrophys 526:A135.

Jones, B.W., Sleep, P.N., and Underwood, D.R. (2006) Habitability of known exoplanets based on measured stellar properties. Astrophys J 649:1010-1019.

Joshi, M.M. and Haberle, R.M. (2012) Suppression of the water ice and snow albedo feedback on planets orbiting red dwarf stars and the subsequent widening of the habitable zone. Astrobiology 12:3-8.

Kaltenegger, L., Udry, S., and Pepe, F. (2011) A habitable planet around HD 85512? arXiv:1108.3561.

Kasting, J.F. (1988) Runaway and moist greenhouse atmospheres and the evolution of Earth and Venus. Icarus 74:472-494.

Kasting, J.F., Whitmore, D.P., and Reynolds, R.T (1993) Habitable zones around main sequence stars. Icarus 101:108-128.

Kite, E.S., Manga, M., and Gaidos, E. (2009) Geodynamics and rate of volcanism of massive Earth-like planets. Astrophys I 700:1732-1749.

Kitzmann, D., Patzer, A.B.C, von Paris, P., Godolt, M., Stracke, B., Gebauer, S., Grenfell, J.L., and Rauer, H. (2010) Clouds in the atmospheres of extrasolar planets I: climatic effects of multi-layered clouds for Earth-like planets and implications of habitable zones. Astron Astrophys 511:A66.

Kopparapu, R., Ramirez, R., Kasting, J.F., Eymet, V., Robinson, T.D., Mahadevan, S., Terrien, R.C., Domagal-Goldman, S., Meadows, V., and Deshpande, R. (2013) Habitable zones around main-sequence stars: new estimates. Astrophys J 765, doi:10.1088/0004-637X/765/2/131.

Lammer, H., Bredehöft, J.H., Coustenis, A., Khodachenko, M.L., Kaltenegger, L., Grasset, O., Prieur, D., Raulin, F., Ehrenfruend, P., Yamauchi, M., Wahlund, J.-E., Griebmeier, J.-M., Stangl, G., Cockell, C.S., Kulikov, Yu.N., Grenfell, J.L., and Rauer, H. (2009) What makes a planet habitable? Astron Astrophys 17:181-249.

Laskar, J., Correia, A.C.M., Gastineau, M., Joutel, F., Levard, B., and Robutel, P. (2004) Long term evolution and chaotic diffusion of the insolation quantities of Mars. Icarus 170:343-364.

Laughlin, G. (1997) The end of the main sequence. Astrophys I 482:420-432.

Lineweaver, C.H. and Davis, T.M. (2002) Does the rapid appearance of life on Earth suggest that life is common in the Universe? Astrobiology 2:293-304.

Lineweaver, C.H., Fenner, Y., and Gibson, B.K. (2004) The galactic habitable zone and the age distribution of complex life in the Milky Way. Science 303:59-62.

McDougall, I., Brown, F., and Fleagle, J.G. (2005) Stratigraphic placement and age of modern humans from Kibish, Ethiopia. Nature 433:733-736.

Nebojsa, D. (2004) Advanced Astrophysics, Cambridge University Press, Cambridge, UK.

O'Malley-James, J.T., Greaves, J.S., Raven, J.A., and Cockell, C.S. (2012) Swansong biospheres: refuges for life and novel microbial biospheres on terrestrial planets near the end of their 
habitable lifetimes. International Journal of Astrobiology 12:99112.

O'Neill, C. and Lenardic, A. (2007) Geological consequences of super-sized Earths. Geophys Res Lett 34:L19204.

Pappalardo, R.T. (2010) Seeking Europa's ocean. Galileo's Medicean moons: their impact on 400 years of discovery. Proceedings IAU Symposium 269:101-114.

Pepe, F., Lovis, C., Ségransan, D., Benz, W., Bouchy, F., Dumusque, X., Mayor, M., Queloz, D., Santos, N.C., and Udry, S. (2011) The HARPS search for Earth-like planets in the habitable zone: I. Very low-mass planets around HD 20794, HD 85512, and HD 192310. Astron Astrophys 534, doi:10.1051/ 0004-6361/201117055.

Pierrehumbert, R. (2010) Principals of Planetary Climate, $1^{\text {st }}$ ed., Cambridge University Press, Cambridge, UK.

Pierrehumbert, R. and Gaidos, E. (2011) Hydrogen greenhouse planets beyond the habitable zone. Astrophys I 734:L13.

Planetary Habitability Laboratory. (2012) The Habitable Exoplanets Catalog. Available online at http://phl.upr.edu/ projects/habitable-exoplanets-catalog.

Raymond, S.N., Scalo. J., and Meadows, V.S. (2007) A decreased probability of habitable planet formation around low-mass stars. Astrophys I 669:606-614.

Sackmann, I.-J., Boothroyd, A.I., and Kraemer, K.E. (1993) Our Sun. III. Present and future. Astrophys I 418:457-468.

Seager, S. (2013) Exoplanet habitability. Science 340:577-581.

Schulze-Makuch, D., Méndez, A., Fairén, A.G., von Paris, P., Turse, C., Boyer, G., Davila, A.F., Resendes de Sousa António, M., Catling, D., and Irwin, L.N. (2011) A two-tiered approach to assessing the habitability of exoplanets. Astrobiology 11:1041-1052.

Selsis, F., Kasting, J.F., Levard, B., Paillet, J., Ribas, I., and Delfosse, X. (2007) Habitable planets around the star Gliese 581? Astron Astrophys 476:1373-1387.

Sleep, N.H. and Zahnle, K. (2001) Carbon dioxide cycling and implications for climate on ancient Earth. L Geophys Res 106:1373-1399.

Spiegel, D.S. and Turner, E.L. (2012) Bayesian analysis of the astrobiological implications of life's early emergence of Earth. Proc Natl Acad Sci USA 109:395-400.

Stamenković, V., Noack, L., Breuer, D., and Spohn, T. (2012) The influence of pressure-dependent viscosity on the thermal evolution of super-Earths. Astrophys J 748, doi:10.1088/ 0004-637X/748/1/41.

Szathmáry, E. and Maynard-Smith, J. (1995) The major evolutionary transitions. Nature 374:227-232.

Tarter, J.C., Backus, P.R., Mancinelli, R.L., Aurnou, J.M., Backman, D.E., Basri, G.S., Boss, A.P., Clarke, A., Deming, D., Doyle, L.R., Feigelson, E.D., Freund, F., Grinspoon, D.H., Haberle, R.M., Hauck, S.A., Heath, M.J., Henry, T.J., Hol- lingsworth, J.L., Joshi, M.M., Kilston, S., Liu, M.C., Meikle, E., Reid, I.N., Rothschild, L.J., Scalo, J., Segura, A., Tang, C.M., Tiedje, J.M., Turnbull, M.C., Walkowicz, L.M., Weber, A.L., and Young, R.E. (2007) A reappraisal of the habitability of planets around M dwarf stars. Astrobiology 7:30-65.

Tuomi, M. (2011) Bayesian re-analysis of the radial velocities of Gliese 581. Astron Astrophys 528:L5.

Tuomi, M., Anglada-Escudé, G., Gerlach, E., Jones, H.R.A., Reiners, A., Rivera, E.J., Vogt, S.S., and Butler, R.P. (2013) Habitable-zone super-Earth candidate in a six-planet system around the K2.5V star HD 40307. Astron Astrophys 549, doi:10.1051/0004-6361/201220268.

Valencia, D., O'Connell, R.J., and Sasselov, D.D. (2007) Inevitability of plate tectonics on super Earths. Astrophys J 670:L45L48.

Vogt, S.S., Butler, R.P., and Haghighipour, N. (2012) GJ 581 update: additional evidence for a Super-Earth in the habitable zone. Astronomische Nachrichten 333:1-15.

von Bloh, W., Bounama, C., Cuntz, M., and Franck, S. (2007) The habitability of super-Earths in Gliese 581. Astron Astrophys 476:1365-1371.

Waltham, D. (2011) Anthropic selection and the habitability of planets orbiting M and K dwarfs. Icarus 215:518-521.

Watson, A.J. (2008) Implications of an anthropic model of evolution for emergence of complex life and intelligence. Astrobiology 8:1-12.

Wordsworth, R.D., Forget, F., Selsis, F., Madeleine, J.-B., Millour, E., and Eymet, V. (2010) Is Gliese 581d habitable? Some constraints from radiative-convective climate modeling. Astron Astrophys 522:A52.

Zsom, A., Kaltenegger, L., and Goldblatt, C. (2012) A 1D microphysical cloud model for Earth, and Earth-like exoplanets: liquid water and water ice clouds in the convective troposphere. Icarus 221:603-616.

Address correspondence to: Andrew J. Rushby

School of Environmental Sciences University of East Anglia

Norwich

Norfolk

NR4 7TJ

UK

E-mail: andrew.rushby@uea.ac.uk

Submitted 12 October 2012

Accepted 9 June 2013 
This article has been cited by:

1. Heller René, Armstrong John. 2014. Superhabitable Worlds. Astrobiology 14:1, 50-66. [Abstract] [Full Text HTML] [Full Text PDF] [Full Text PDF with Links] 